\title{
CHARACTERIZING GENERIC GLOBAL RIGIDITY
}

\author{
STEVEN J. GORTLER, ALEXANDER D. HEALY, AND DYLAN P. THURSTON
}

\begin{abstract}
A $d$-dimensional framework is a graph and a map from its vertices to $\mathbb{E}^{d}$. Such a framework is globally rigid if it is the only framework in $\mathbb{E}^{d}$ with the same graph and edge lengths, up to rigid motions. For which underlying graphs is a generic framework globally rigid? We answer this question by proving a conjecture by Connelly, that his sufficient condition is also necessary: a generic framework is globally rigid if and only if it has a stress matrix with kernel of dimension $d+1$, the minimum possible.

An alternate version of the condition comes from considering the geometry of the lengthsquared mapping $\ell$ : the graph is generically locally rigid iff the rank of $\ell$ is maximal, and it is generically globally rigid iff the rank of the Gauss map on the image of $\ell$ is maximal.

We also show that this condition is efficiently checkable with a randomized algorithm, and prove that if a graph is not generically globally rigid then it is flexible one dimension higher.
\end{abstract}

\section{INTRODUCTION}

In this paper we characterize those generic frameworks which are globally rigid in $d$ dimensional Euclidean space. We do this by proving a conjecture of Connelly [10], who described a sufficient condition for generic frameworks to be globally rigid, and conjectured that this condition was necessary. As this condition depends only on the graph and the dimension $d$, and not on the specific (generic) framework of that graph, we can conclude that generic global rigidity in $\mathbb{E}^{d}$ is a property of a graph. We further show that this property can be checked in probabilistic polynomial time.

Global rigidity has applications in chemistry, where various technologies measure interatomic distances. From this data one may try to infer the geometry of the configuration [11, inter alia]. This inference is only well posed if the associated framework is globally rigid. Moreover, testing for generic global rigidity can be used as part of a divide-and-conquer strategy for this inference [18]. Similar problems arise in the field of sensor networks [7, inter alia].

\subsection{Definitions and results.}

Definition 1.1. A graph $\Gamma$ is a set of $v$ vertices $\mathcal{V}(\Gamma)$ and $e$ edges $\mathcal{E}(\Gamma)$, where $\mathcal{E}(\Gamma)$ is a set of two-element subsets of $\mathcal{V}(\Gamma)$. We will typically drop the graph $\Gamma$ from this notation. A configuration of $\Gamma$ in $\mathbb{E}^{d}$ is a mapping from $\mathcal{V}(\Gamma)$ to Euclidean space $\mathbb{E}^{d}$. A framework $\rho$ in $\mathbb{E}^{d}$ is a graph $\Gamma$ together with a configuration of $\Gamma$ in $\mathbb{E}^{d}$; we will also say that $\rho$ is a framework of $\Gamma$. Let $C^{d}(\Gamma)$ denote the space of frameworks with a given graph $\Gamma$ and dimension $d$. For $\rho \in C^{d}(\Gamma)$ and $u \in \mathcal{V}(\Gamma)$, let $\rho(u)$ denote the image of $u$ under the configuration of $\rho$. For a given graph $\Gamma$ and dimension $d$, the length-squared function $\ell: C^{d}(\Gamma) \rightarrow \mathbb{R}^{e}$ is the function assigning to each edge of $\Gamma$ its squared edge length in the framework. In particular, the component of $\ell(\rho)$ in the direction of an edge $\{u, w\}$ is $|\rho(u)-\rho(w)|^{2}$.

1991 Mathematics Subject Classification. 05C62; 14P99. 
Definition 1.2. A framework $\rho$ in $\mathbb{E}^{d}$ is congruent to another framework if they are related by an element of the group $\operatorname{Eucl}(d)$ of rigid motions of $\mathbb{E}^{d}$ (rotations, reflections, and translations). We say that $\rho$ is globally rigid if $\rho$ is the only framework of $\Gamma$ in $\mathbb{E}^{d}$ with the same edge lengths, up to congruence. Equivalently, $\rho$ is globally rigid iff $\ell^{-1}(\ell(\rho)) / \operatorname{Eucl}(d)$ consists of $[\rho]$, the congruence class of frameworks which contains $\rho$.

Definition 1.3. A framework $\rho \in C^{d}(\Gamma)$ is locally rigid if there exists a neighborhood $U$ of $\rho$ in $C^{d}(\Gamma)$ such that $\rho$ is the only framework in $U$ with the same set of edge lengths, up to congruence; equivalently, $[\rho]$ is isolated in $\ell^{-1}(\ell(\rho)) / \operatorname{Eucl}(d)$.

(This property is usually just called rigidity but we will use term local rigidity in this paper to make explicit its distinction from global rigidity.)

Definition 1.4. A framework is generic if the coordinates of its configuration do not satisfy any non-trivial algebraic equation with rational coefficients.

We first deal with graphs with very few vertices. Asimow and Roth proved that a generic framework $\rho$ in $\mathbb{E}^{d}$ of a graph $\Gamma$ with $d+1$ or fewer vertices is globally rigid if $\Gamma$ is a complete graph (i.e., a simplex), otherwise it is not even locally rigid [1, Corollary 4]. Therefore in the rest of the paper we may assume that our graph has $d+2$ or more vertices. In particular, this implies that a generic framework does not lie in a proper affine subspace of $\mathbb{E}^{d}$.

Since $\operatorname{Eucl}(d)$ acts freely on frameworks that do not lie in a proper affine subspace of $\mathbb{E}^{d}$, for such a framework $\rho$, the fiber $\ell^{-1}(\ell(\rho))$ always has dimension at least $\operatorname{dim}(\operatorname{Eucl}(d)$ ) (which is $\left.\left(\begin{array}{c}d+1 \\ 2\end{array}\right)\right)$. In particular, a generic framework with at least $d+1$ vertices satisfies this condition. The intuition behind the following definition and theorem is that the dimension of a generic fiber of an algebraic map $f$ is the same as the kernel of the Jacobian $d f_{x}$ of $f$ at a generic point $x$, and for a generic locally rigid framework the kernel of $d f_{x}$ only contains the tangents to the action of $\operatorname{Eucl}(d)$.

Definition 1.5. Let $d \ell_{\rho}$ be the rigidity matrix of $\rho$, the Jacobian of $\ell$ at the framework $\rho$; by definition, this is a linear map from $C^{d}(\Gamma)$ to $\mathbb{R}^{e}$. A framework $\rho \in C^{d}(\Gamma)$ of a graph $\Gamma$ with $d+1$ or more vertices is infinitesimally rigid if

$$
\operatorname{rank} d \ell_{\rho}=\operatorname{dim}\left(C^{d}(\Gamma)\right)-\operatorname{dim}(\operatorname{Eucl}(d))=v d-\left(\begin{array}{c}
d+1 \\
2
\end{array}\right) .
$$

Theorem 1.6 (Asimow-Roth [1]). If a generic framework $\rho \in C^{d}(\Gamma)$ of a graph $\Gamma$ with $d+1$ or more vertices is locally rigid in $\mathbb{E}^{d}$, then it is infinitesimally rigid. Otherwise rank $d \ell_{\rho}$ is lower than $v d-\left(\begin{array}{c}d+1 \\ 2\end{array}\right)$.

Since the rank of the linearization an algebraic map is the same (and maximal) at every generic point, local rigidity in $\mathbb{E}^{d}$ is a generic property. (See also Lemma 5.6.) That is, either all generic frameworks in $C^{d}(\Gamma)$ are locally rigid, or none of them are. Thus we can call this condition generic local rigidity in $\mathbb{E}^{d}$ and consider it as a property of the graph $\Gamma$.

We next define some concepts we need to state the condition for generic global rigidity.

Definition 1.7. An equilibrium stress vector of a framework $\rho$ of $\Gamma$ is a real valued function $\omega$ on the (undirected) edges of $\Gamma$ so that for all vertices $u \in \mathcal{V}$,

$$
\sum_{\{w \in \mathcal{V} \mid\{u, w\} \in \mathcal{E}\}} \omega(\{u, w\})(\rho(w)-\rho(u))=0 .
$$


(Note that the all-zero function is an equilibrium stress vector for any $\rho$.) Let $S(\rho)$ be the vector space of equilibrium stress vectors of the framework $\rho$.

Remark 1.8. In many cases, we can interpret (2) as saying that each vertex $u$ is the weighted average of its neighbors, with weights given by $\omega(\{u, w\})$. This interpretation breaks down if $\sum_{w} \omega(\{u, w\})=0$, as happens, for instance, for $K_{5,5}$ in $\mathbb{E}^{3}$. (See Section 3.4.) An alternate interpretation is that if we put springs on the edges of $\Gamma$ with spring constants given by $\omega$, the resulting forces on each vertex balance out. Note, however, that some of the spring constants in the graph are necessarily negative, and the rest position is when the spring is at zero length.

Definition 1.9. An equilibrium stress matrix of a framework $\rho$ of $\Gamma$ is a rearrangement of an equilibrium stress vector into a matrix form, with suitably chosen diagonal entries. More precisely, it is a matrix $\Omega$ indexed by $\mathcal{V} \times \mathcal{V}$ so that

(1) for all $u, w \in \mathcal{V}, \Omega(u, w)=\Omega(w, u)$;

(2) for all $u, w \in \mathcal{V}$ with $u \neq w$ and $\{u, w\} \notin \mathcal{E}, \Omega(u, w)=0$;

(3) for all $u \in \mathcal{V}, \sum_{w \in \mathcal{V}} \Omega(u, w)=0$; and

(4) for all $u \in \mathcal{V}, \sum_{w \in \mathcal{V}} \Omega(u, w) \rho(w)=0$.

For every equilibrium stress vector $\omega$ there is a unique equilibrium stress matrix $\Omega$ so that for $u \neq w$ and $\{u, w\} \in \mathcal{E}, \Omega(u, w)=\omega(\{u, w\})$, and conversely. (This implies that $\Omega(u, u)=$ $-\sum_{w \neq u} \omega(\{u, w\})$.) Thus we will freely convert between equilibrium stress vectors $\omega$ and equilibrium stress matrices $\Omega$. If $\Omega$ is an equilibrium stress matrix for $\rho$, we will also say that $\rho$ satisfies the stress matrix $\Omega$.

Definition 1.10. For $\Omega$ an equilibrium stress matrix, the stress kernel $K(\Omega)$ is the kernel of $\Omega$. It is isomorphic to the space of frameworks of $\Gamma$ in $\mathbb{E}^{1}$ which have $\Omega$ as an equilibrium stress. Let $k(\Omega)$ or simply $k$ be $\operatorname{dim}(K(\Omega))$.

Let $k_{\min }(\Gamma, d)$ or just $k_{\min }$ be the minimal value of $k(\Omega)$ as $\Omega$ ranges over all stress matrices of all generic frameworks in $C^{d}(\Gamma)$. As has been noted by Hendrickson [18, Theorem 2.5], for each fixed generic framework $\rho$, the minimal value of $k(\Omega)$ as $\Omega$ ranges over $S(\rho)$ agrees with this $k_{\text {min }}$. Indeed $k(\Omega)=k_{\text {min }}$ for all generic frameworks $\rho$ and generic $\Omega \in S(\rho)$, suitably defined. (See Lemma 5.8.)

$K(\Omega)$ always contains the subspace spanned by the coordinates of $\rho$ along each axis and the vector $\overrightarrow{1}$ of all 1 's. This corresponds to the fact that any affine image of $\rho$ satisfies all of the equilibrium stress matrices in $S(\rho)$. If $\rho$ does not lie in a proper affine subspace of $\mathbb{E}^{d}$, these vectors are independent and span a $(d+1)$-dimensional space. We have therefore proved the following lemma.

Lemma 1.11. For frameworks of a graph $\Gamma$ with at least $d+1$ vertices, $k_{\min }(\Gamma, d) \geq d+1$.

Definition 1.12. A graph $\Gamma$ has a minimal stress kernel in $\mathbb{E}^{d}$ if $k_{\min }(\Gamma, d)=d+1$.

We can now state Connelly's criterion for generic global rigidity.

Theorem 1.13 (Connelly [10]). If a graph $\Gamma$ with $d+2$ or more vertices has a minimal stress kernel in $\mathbb{E}^{d}$, then all generic frameworks $\rho \in C^{d}(\Gamma)$ are globally rigid.

Our main result is the converse of Connelly's result, which completes the characterization of generic global rigidity. 
Theorem 1.14. If a graph $\Gamma$ with $d+2$ or more vertices does not have a minimal stress kernel in $\mathbb{E}^{d}$, then any generic framework $\rho \in C^{d}(\Gamma)$ is not globally rigid.

The proof of Theorem 1.14 is in Section 2.

Putting these two together, we can also conclude that global rigidity is a generic property.

Corollary 1.15. Either all generic frameworks in $C^{d}(\Gamma)$ of a graph are globally rigid, or none of them are. Thus we can call this condition generic global rigidity in $\mathbb{E}^{d}$ and consider it as a property of a graph.

Since the condition is an algebraic condition, equivalent statements to generic global rigidity in $\mathbb{E}^{d}$ include the following:

- frameworks in a Zariski open subset of $C^{d}(\Gamma)$ are globally rigid; or

- frameworks in a subset of full measure of $C^{d}(\Gamma)$ are globally rigid.

In fact, we can deduce a stronger statement.

Corollary 1.16. If a graph $\Gamma$ with $d+2$ or more vertices does not have a minimal stress kernel in $\mathbb{E}^{d}$, then any infinitesimally rigid framework $\rho \in C^{d}(\Gamma)$ is not globally rigid.

(This corollary can also be proven by combining corollary 1.15 with [8, Proposition 2.4].)

Proof. Let $\rho_{0}$ be an infinitesimally rigid framework. First note that $\rho_{0}$ does not lie in any affine subspace of $\mathbb{E}^{d}$, so by Proposition 2.13 it maps to a smooth point $\left[\rho_{0}\right]$ in the quotient $C^{d}(\Gamma) / \operatorname{Eucl}(d)$. By the inverse function theorem, $\left[\rho_{0}\right]$ has an open neighborhood $N$ that maps injectively into the space of edge lengths. Consider a sequence $\left\{\left[\rho_{i}\right]\right\}$ of classes of generic configurations inside $N$ approaching $\left[\rho_{0}\right]$. By Theorem 1.14, for each $\rho_{i}$ there is an inequivalent configuration $\sigma_{i}$ with the same edge lengths. Because the map $\ell$ is proper on $C^{d}(\Gamma) / \operatorname{Eucl}(d)$ (see Lemma 2.34), the sequence $\left\{\left[\sigma_{i}\right]\right\}$ has an accumulation point $\left[\sigma_{0}\right]$, with $\ell\left(\sigma_{0}\right)=\ell\left(\rho_{0}\right)$ by continuity. Since $\ell$ is injective on $N$, we have $\left[\sigma_{i}\right] \notin N$ and so $\left[\sigma_{0}\right] \notin N$; thus $\sigma_{0}$ is the desired framework with the same edge lengths as $\rho_{0}$ but not congruent to $\rho_{0}$.

Note that there is no hope for a similar strengthening of Theorem 1.13; global rigidity can fail at non-generic points in any number of ways, for instance if there is a transverse self-intersection of the image space $\ell\left(C^{d}(\Gamma)\right)$.

As a corollary of Theorems 1.13 and 1.14, we find an efficient algorithm for checking for generic global rigidity.

Theorem 1.17. There is a polynomial-time randomized algorithm for checking for generic global rigidity in $\mathbb{E}^{d}$.

Essentially, you take random a framework $\rho$ in $C^{d}(\Gamma)$, using sufficiently large random numbers as coordinates, and a random equilibrium stress matrix $\Omega$ of $\rho$. Then with high probability, $\operatorname{dim}(\operatorname{ker}(\Omega))=k_{\min }$. In Section 5 we prove a more precise version of this statement (Theorem 5.10), in particular giving bounds on "sufficiently large".

One further result, arising from similar considerations involved in the proof of Theorem 1.14, is the following:

Theorem 1.18. If a graph $\Gamma$ is not generically globally rigid in $\mathbb{E}^{d}$, then any generic framework $\rho$ in $C^{d}(\Gamma)$ can be connected to some incongruent framework $\sigma$ in $C^{d}(\Gamma)$ by a path of frameworks of $\Gamma$ in $\mathbb{E}^{d+1}$ with constant edge lengths.

Theorem 1.18 is proved in Section 6. 
1.2. Relationship to previous work. The problem of $d$-embeddability, determining if there is a framework of a graph in $\mathbb{E}^{d}$ with a given set of (integer) edge lengths, was shown to be strongly NP-hard by Saxe [26]. Saxe also showed that the problem of determining global rigidity is strongly NP-hard. The proof starts with the observation that a 1-dimensional framework of a graph formed of $n$ vertices connected in a cycle is a solution to the partition problem on the edge lengths.

But there has been some hope that global rigidity can be characterized and efficiently tested if one suitably restricts the input to avoid certain coincidences (like the coincidence that there be two solutions to a partition problem that has at least one solution). In particular, one strong way of restricting the problem is to assume that the original framework is generic. For example, it is easy to see that a generic framework of a graph is globally rigid in $\mathbb{E}^{1}$ iff the graph is 2-connected.

Hendrickson [18] describes the condition of Theorem 1.13 ascribed to Connelly (CC for short), for generic frameworks of a graph to be globally rigid in $\mathbb{E}^{d}$. Connelly proved the sufficiency of this condition [10]. Connelly also conjectured that CC was a necessary condition, our Theorem 1.14.

Hendrickson also gave [17] two necessary conditions (HC for short), for generic frameworks of $\Gamma$ to be globally rigid in $\mathbb{E}^{d}$. Namely, $\Gamma$ must be $(d+1)$-connected and deleting any edge of $\Gamma$ must leave a generically locally rigid graph. A combinatorial result of Jackson and Jordán [19] effectively shows that for $d=2, \mathrm{CC}=\mathrm{HC}$, and so both conditions are necessary and sufficient. They later gave a direct proof of the sufficiency of HC for $d=2$ [20]. Moreover in 2 dimensions, generic local rigidity and therefore generic global rigidity can be characterized combinatorially [21] and deterministically solved for efficiently [23, inter alia].

Connelly showed [9] that $\mathrm{HC}$ are not sufficient to show that a generic framework is globally rigid for $d \geq 3$; specifically, he showed that the complete bipartite graph $K_{5,5}$, despite satisfying $\mathrm{HC}$, is not globally rigid in 3 dimensions for some generic frameworks. Similar results hold for larger bipartite graphs in higher dimensions. These appear to be the only known examples of such graphs.

(See Sections 3.2 and 3.3 for more on graphs that fail HC, and Section 3.4 for more on $\left.K_{5,5} \cdot\right)$

1.3. Structure of the paper. We prove the main result in Section 2 and give a few examples of the relevant spaces in Section 3.

In Section 4 we give an alternate characterization of generic global rigidity in terms of the shared stress kernel (the intersection of the kernels of all stress matrices) or, alternatively, the rank of the Gauss map. (The Gauss map is the map that takes a smooth point of an algebraic variety to its tangent space, considered as a point in an appropriate Grassmannian.) We also relate the theorem to the more general algebraic geometry setting of secant varieties and Zak's theorem.

We then show that the condition of generic global rigidity can be checked in randomized polynomial time in Section 5, following remarks of Hendrickson [17, 18].

In Section 6 we prove Theorem 1.18.

Throughout this paper, we attempt to give enough details for readers who are not very familiar with algebraic geometry, while at the same time indicating how the more abstract arguments work. 


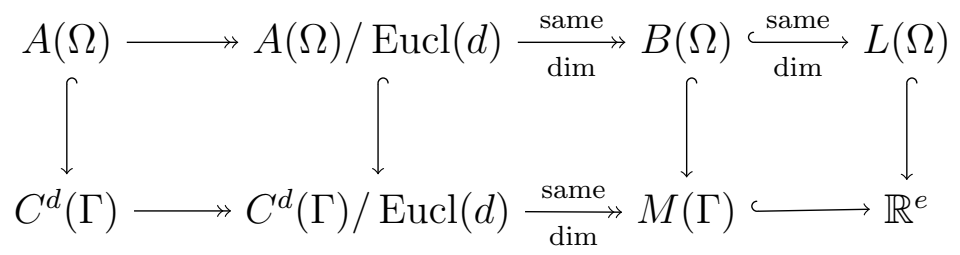

FiguRE 1. The main spaces involved in the proof of Theorem 1.14

Acknowledgements. We would like to thank Robert Connelly, Daniel Freedman, Yael Karshon, J. M. Landsberg, Amit Singer, Ileana Streinu and an anonymous referee for helpful conversations and suggestions. AH was supported by NSF grant CCR-0205423 and a Sandia fellowship. DPT was supported by a Sloan research fellowship.

\section{PRoOf OF MAIN THEOREM}

Our basic strategy for the proof of Theorem 1.14 is similar in spirit to the approach used by Hendrickson [17] to show the necessity of what he called "redundant rigidity" for a generic global rigidity in $\mathbb{E}^{d}$. Given a graph $\Gamma$ which does not have a minimal stress kernel in $\mathbb{E}^{d}$, we construct spaces $X$ and $Y$ and a map $f: X \rightarrow Y$ so that the preimages in $X$ of a point in $Y$ correspond to incongruent frameworks with the same edge lengths. We then show that the "degree mod-two" of this map is well defined and equal to 0 . This degree is equal to the number of preimages at a regular value, modulo 2. Thus for such a map, any regular value in the image must have at least one more preimage, which represents a distinct framework in $C^{d}(\Gamma)$ with the same edge lengths, thus contradicting global rigidity in $\mathbb{E}^{d}$. To guarantee a well defined degree, we will not have the luxury of the domain being a compact manifold. Rather the domain will be a smooth space with singularities of co-dimension 2 or greater, and $f$ will be a proper map. (See Definition 2.33 and Theorem 2.35.)

The main spaces involved in the proof are summarized in Figure 1, as we now explain. We start with the map $\ell$ from $C^{d}(\Gamma)$ to $\mathbb{R}^{e}$, and factor it through the quotient by the Euclidean group, followed by the surjection onto its image $M(\Gamma) \subset \mathbb{R}^{e}$. The spaces $C^{d}(\Gamma) / \operatorname{Eucl}(d)$ and its image $M(\Gamma)$ have the same dimension by local rigidity.

We next take any generic $\rho$ and pick an equilibrium stress $\Omega$ for $\rho$ which is generic in a suitable sense, and consider the space $A(\Omega)$ of all frameworks satisfying $\Omega$. As motivation for this, recall that Connelly's proof of Theorem 1.13 first shows that for any generic framework $\rho$ and a global flex $\sigma$ of it, the map $\ell$ agrees on neighborhoods of $\rho$ and $\sigma$ [10, Proposition 3.3]. In particular, the tangent space to $M(\Gamma)$ at $\rho$ and $\sigma$ agree, so any equilibrium stress for $\rho$ is also an equilibrium stress for $\sigma$. (Compare Lemma 2.21.) Thus $\sigma$ must lie in $A(\Omega)$, for any $\Omega \in S(\rho)$. So we may as well look for a global flex within such a space $A(\Omega)$.

We define the domain of our map to be $A(\Omega) / \operatorname{Eucl}(d)$. In Proposition 2.13, we show that if the stress kernel of $\Omega$ is of dimension greater than $d+1$, then this domain has singularities of codimension at least 2. This is based on recognizing $A(\Omega)$ as $K(\Omega)^{d}$, where $K(\Omega)$ is the stress kernel as in Definition 1.10.

We also consider the image of $A(\Omega)$ under $\ell$, denoted $B(\Omega) \subset M(\Gamma)$. Again, by local rigidity, the quotient $A(\Omega) / \operatorname{Eucl}(d)$ and $B(\Omega)$ have the same dimension. If we view the equilibrium stress vector as defining a hyperplane in $\mathbb{R}^{e}$, we can then alternately view $B(\Omega)$ as the contact locus of the corresponding equilibrium stress $\omega$ : the set of points in $M(\Gamma)$ 
whose tangent space is contained in the hyperplane defined by $\omega$. A fundamental theorem of algebraic geometry says that a generic contact locus is flat: it is contained in a linear space $L(\Omega)$ of the same dimension. (See Proposition 2.20.) The space $L(\Omega)$ serves as the range.

Our map $\bar{\ell}$ is the restriction of $\ell$ to this domain and range. Since the singularities of the domain have codimension at least $2, \bar{\ell}$ will have a well-defined degree. The final step in the proof is that this degree is 0 , which is guaranteed by noticing that any point in $\mathbb{R}^{e}$ with at least one negative coordinate is not in the image of $\ell$.

If a generic framework in $C^{d}(\Gamma)$ is not locally rigid, then it is clearly not globally rigid, in which case our proof is done. So, in what follows, we will assume that the framework $\rho \in C^{d}(\Gamma)$ is locally rigid and has at least $d+2$ vertices, and so by Theorem 1.6 , rank $d \ell_{\rho}=$ $v d-\left(\begin{array}{c}d+1 \\ 2\end{array}\right)$.

2.1. Algebraic geometry preliminaries. We start with some preliminaries about semialgebraic sets from real algebraic geometry, somewhat specialized to our particular case. For a general reference, see, for instance, the book by Bochnak, Coste, and Roy [5].

Definition 2.1. An affine, real algebraic set or variety $V$ defined over a field $\mathbf{k}$ contained in $\mathbb{R}$ is a subset of $\mathbb{R}^{n}$ that is defined by a set of algebraic equations with coefficients in $\mathbf{k}$. It is irreducible if it is not the union of two proper algebraic subsets defined over $\mathbb{R}$. An algebraic set has a dimension $\operatorname{dim}(V)$, which we will define as the largest $t$ for which there is an open (Euclidean) subset of $V$ isomorphic to $\mathbb{R}^{t}$.

A point $x$ of an irreducible algebraic set $V$ is smooth if it has a neighborhood that is smoothly isomorphic to $\mathbb{R}^{\operatorname{dim}(V)}$; in this case there is a well-defined tangent space $T_{x}(V)$. (Note that there may be points with neighborhoods isomorphic to $\mathbb{R}^{n}$ for some $n<\operatorname{dim}(V)$; we do not consider these points to be smooth.)

Definition 2.2. A semi-algebraic set $S$ over $\mathbf{k}$ is a subset of $\mathbb{R}^{n}$ defined by algebraic equalities and inequalities with coefficients in $\mathbf{k}$; alternatively, it is the image of an algebraic set (defined only by equalities) under an algebraic map with coefficients in $\mathbf{k}$. A semi-algebraic set has a well defined (maximal) dimension $t$.

The Zariski closure of $S$ is the smallest algebraic set over $\mathbb{R}$ containing it. (Loosely speaking, we can get an algebraic set by keeping all algebraic equalities and dropping the inequalities. We may need to enlarge the field to cut out the smallest algebraic set containing $S$ but a finite extension will always suffice.) We call $S$ irreducible if its Zariski closure is irreducible. (We chose here to define the Zariski closure as well as the notion of irreducibility using the field $\mathbb{R}$ instead of $\mathbb{Q}$ or $\mathbf{k}$ in order to avoid complications in the proof of Proposition 2.18 below.) An irreducible semi-algebraic set $S$ has the same dimension as its Zariski closure.

A point on $S$ is smooth if it has a neighborhood in $S$ smoothly isomorphic to $\mathbb{R}^{\operatorname{dim}(S)}$. We denote by $\operatorname{smooth}(S)$ the locus of smooth points of $S$. It too is semi-algebraic. We call $S$ itself $\operatorname{smooth}$ if $\operatorname{smooth}(S)=S$.

Suppose that $S$ is a smooth irreducible semi-algebraic set. Let $\phi$ be a non-zero algebraic function on $S$. Its zero set is stratified as a union of a finite number of smooth manifolds each with dimension less than $\operatorname{dim}(S)$, so (since $S$ is smooth) the complement of the zero set (i.e., $\{x \in S \mid \phi(x) \neq 0\}$ ) is open and dense in $S$.

Definition 2.3. The image of $\ell$ is called the measurement set and denote by $M(\Gamma)$ or just $M$. It is a semi-algebraic set defined over $\mathbb{Q}$. 
Lemma 2.4. The semi-algebraic set $M$ is irreducible.

Proof. $M$ is the image of $C^{d}(\Gamma)$ under a polynomial map. Since $C^{d}(\Gamma)$ is irreducible and the image of an irreducible variety is irreducible, it follows that $M$ is irreducible.

The dimension of $M$ is the rank of $d \ell_{\rho}$ at any generic configuration $\rho$ (and is $v d-\left(\begin{array}{c}d+1 \\ 2\end{array}\right)$ when the graph is generically locally rigid in $\mathbb{E}^{d}$ ). More precisely, the tangent space to $M$ for generic $\rho$ is the span of the image of $d \ell_{\rho}$.

We next define genericity in larger generality and give some basic properties.

Definition 2.5. A point in a (semi-)algebraic set $V$ defined over $\mathbf{k}$ is generic if its coordinates do not satisfy any algebraic equation with coefficients in $\mathbf{k}$ besides those that are satisfied by every point on $V$.

Almost every point in an irreducible semi-algebraic set $V$ is generic.

Note that the defining field might change when we take the Zariski closure. However, this does not matter for the purposes of genericity. More formally, if $\mathbf{k}$ is a finite algebraic extension of $\mathbb{Q}$ and $x$ is a generic point in an irreducible semi-algebraic set $S$ defined over $\mathbf{k}$, then $x$ is also generic in the Zariski closure of $S$ over an appropriate field. This follows from a two step argument. First, an elementary argument from Galois theory proves that the (real) Zariski closure $V$ of $S$ can be defined using polynomials over a field $\mathbf{k}^{\prime}$ which is some finite extension of $\mathbf{k}$. Second, another elementary argument from Galois theory proves that if a point $x$ satisfies an algebraic equation with coefficients in $\mathbf{k}^{\prime}$ that is non-zero over $V$, then $x$ must also satisfy some algebraic equation with coefficients in $\mathbf{k}$ that is non-zero over $V$.

The following proposition is standard:

Proposition 2.6. Every generic point of a (semi-)algebraic set is smooth.

Lemma 2.7. Let $V$ and $W$ be irreducible semi-algebraic sets and $f: V \rightarrow W$ be a surjective algebraic map, all defined over $\mathbf{k}$. Then if $x_{0} \in V$ is generic, $f\left(x_{0}\right)$ is generic inside $W$.

Proof. Consider any non-zero algebraic function $\phi$ on $W$ defined over $\mathbf{k}$. Then $x \mapsto \phi(f(x))$ is a function on $V$ that is not identically zero. Thus if $x_{0}$ is a generic point in $V, \phi\left(f\left(x_{0}\right)\right) \neq 0$. Since this is true for all $\phi$, it follows that $f\left(x_{0}\right)$ is generic.

Thus, instead of speaking of a generic configuration in $C^{d}(\Gamma)$, we may speak of a generic point on $M$ : if a configuration $\rho$ is generic in $C^{d}(\Gamma)$, then $\ell(\rho)$ is generic in $M$.

Lemma 2.8. Let $\mathbf{k}$ be a finite algebraic extension of $\mathbb{Q}$. Let $V$ and $W$ be irreducible semialgebraic sets with $V$ smooth, and let $f: V \rightarrow W$ be a surjective algebraic map, all defined over $\mathbf{k}$. Then if $y_{0} \in W$ is generic, there is a point in $f^{-1}\left(y_{0}\right)$ that is generic in $V$.

Proof. Let $\phi$ be a non-zero algebraic function on $V$ defined over $\mathbf{k}$. We start by showing there is a point $x \in f^{-1}\left(y_{0}\right)$ so that $\phi(x) \neq 0$. Consider the semi-algebraic set $X_{\phi}:=\{x \in$ $V \mid \phi(x) \neq 0\}$. This is dense in $V$ due to irreducibility and smoothness, so its image $f\left(X_{\phi}\right)$ is dense in $W$. Therefore $Y_{\phi}:=W \backslash f\left(X_{\phi}\right)$ is a semi-algebraic subset of $W$ that is nowhere dense. It follows that there is a non-zero algebraic function $\psi$ on $W$ defined over $\mathbf{k}$ that vanishes when restricted to $Y_{\phi}$. But then since $y_{0}$ is generic, $\psi\left(y_{0}\right) \neq 0$, which means that $y_{0}$ is in the image of $X_{\phi}$, so there is an $x \in f^{-1}\left(y_{0}\right)$ so that $\phi(x) \neq 0$, as desired.

Let $Z_{\phi}=\left\{x \in f^{-1}\left(y_{0}\right) \mid \phi(x)=0\right\}$. We have shown $Z_{\phi}$ is a proper subset of $f^{-1}\left(y_{0}\right)$ for all non-zero algebraic functions $\phi$ on $V$. It follows that for any finite collection of $\phi_{i}$, the union of the $Z_{\phi_{i}}$ is still a proper subset of $f^{-1}\left(y_{0}\right)$ (as we can consider the product of the $\phi_{i}$ ). 
But there are only countably many possible $\phi$ overall, and a countable union of algebraic subsets covers an algebraic set iff some finite collection of them do. (Proof: this is true for each irreducible component, as a proper algebraic subset has measure zero, and there are only finitely many irreducible components.) Thus the union of the $Z_{\phi}$ do not cover $f^{-1}\left(y_{0}\right)$, i.e., there is a generic point in $f^{-1}\left(y_{0}\right)$.

Lemma 2.9. Let $V \subset W$ be an inclusion of real algebraic varieties, where $W$ (but not $V$ ) is defined over $\mathbb{Q}$. Suppose that $V$ is smooth and irreducible and that it has one point $y$ which is generic in $W$ (over $\mathbb{Q})$. Then the points in $V$ which are generic in $W$ are dense in $V$.

Note that in this lemma the points we consider are different from the points that are generic in $V$, since $V$ is not defined using only the rationals. (If $V$ were defined using the rationals and were properly contained in $W$, then it would have no points which were generic in $W$ : any rational equation defining $V$ would by definition vanish for all $x \in V$.)

Proof. Let $\phi$ be a non-zero algebraic function on $W$ defined over $\mathbb{Q}$. Consider the semialgebraic set $X_{\phi}:=\{x \in V \mid \phi(x) \neq 0\}$. This is non empty due to our assumption about the point $y$. Thus $X_{\phi}$ is open and dense in $V$, due to irreducibility and smoothness. The set of points in $V$ which are generic in $W$ is defined as the intersection of the $X_{\phi}$ as $\phi$ ranges over the countable set of possible $\phi$. Since $V$ is a Baire space, such a countable intersection of open and dense subsets must itself be a dense subset.

2.2. The domain. To construct the domain, we start by considering stress satisfying frameworks.

Definition 2.10. Let $\Omega$ be an equilibrium stress matrix of some framework in $C^{d}(\Gamma)$. The space of stress satisfiers $A(\Omega)$ is the space of all $d$-dimensional frameworks that satisfy $\Omega$ :

$$
A(\Omega):=\left\{\sigma \in C^{d}(\Gamma) \mid \Omega \in S(\sigma)\right\} .
$$

Lemma 2.11. The space $A(\Omega)$ is isomorphic to $K(\Omega)^{d}$ and has dimension $k(\Omega) d$. In particular, if $\Omega \in S(\rho)$, then $A(\Omega)$ contains the space $\operatorname{Aff}(\rho)$ of affine images of $\rho$.

Proof. Since Equation (2) is an equation for each coordinate separately, a framework satisfies an equilibrium stress iff each of its projections on the coordinate axes does. But $K(\Omega)$ is isomorphic to the space of 1-dimensional frameworks of $\Gamma$ that have $\Omega$ as an equilibrium stress, so it follows that $A(\Omega)$ is $K(\Omega)^{d}$. The remaining statements are immediate.

We now study the quotient $A(\Omega) / \operatorname{Eucl}(d)$, which, for an appropriate choice of $\Omega$, will be the domain of our main map. If $\Gamma$ is generically locally rigid and $A(\Omega)$ includes some framework generic in $C^{d}(\Gamma)$, this space is of dimension $k(\Omega) d-\left(\begin{array}{c}d+1 \\ 2\end{array}\right)$. We need to understand the singularities of this quotient, and in particular show that they have codimension at least 2 in appropriate cases (so that we can apply Corollary 2.36). We will reduce the question to the following lemma.

Lemma 2.12. If $n>d$, the quotient space $\left(\mathbb{E}^{d}\right)^{n} / \operatorname{Eucl}(d)$ (i.e., $n$-tuples of points in $\mathbb{E}^{d}$, quotiented by $\operatorname{Eucl}(d)$ acting diagonally) is a smooth stratified space with singularities of codimension $n-d$ and higher. Furthermore, the singularities occur at classes of points in $\mathbb{E}^{d}$ that lie inside a proper affine subspace of $\mathbb{E}^{d}$.

Here a smooth stratified space is, loosely speaking, a space which is decomposed into smooth manifolds of differing dimensions, limiting onto each other in a nice way. For instance, 
any semi-algebraic set, with any type of singularities, has the structure of a smooth stratified space. For a formal definition and discussion, see [24].

Proof. In general, the quotient of a variety by a group acting properly is stratified by conjugacy classes of stabilizers [24, Corollary 4.3.11]. In particular the points with trivial stabilizer form a manifold. Let us therefore identify the points in $\left(\mathbb{E}^{d}\right)^{n}$ where the stabilizer is non-trivial. The key observation is that an $n$-tuple is stabilized by a non-trivial Euclidean transform if and only if the dimension of its affine span is less than $d$. For example, if all points lie in a hyperplane of dimension $d-1$, then they are stabilized by the reflection across that plane. In general, if the affine span of the $n$-tuple is codimension $r$, the stabilizer is isomorphic to the orthogonal group in $r$ dimensions, $O(r)$.

The lowest codimension case is when $r=1$, in which case the stabilizer is $O(1) \simeq \mathbb{Z} / 2$. To compute the codimension of these $n$-tuples with $r=1$ in $\left(\mathbb{E}^{d}\right)^{n}$, pick $d$ out of the $n$ points that span the subspace; these points are unrestricted. The remaining $n-d$ points must lie in that subspace, providing one constraint for each point, so these $n$-tuples have codimension $n-d$. In general, a similar count shows that $n$-tuples with a stabilizer of $O(r)$ are of codimension $r(n-d+r-1)$ in $\left(\mathbb{E}^{d}\right)^{n}$.

Inside the quotient $\left(\mathbb{E}^{d}\right)^{n} / \operatorname{Eucl}(d)$, the codimension of the singular set is decreased by the dimension of the stabilizer. Thus points with stabilizer $O(1)$ still have codimension $n-d$ in the quotient, and in general the the codimension in the quotient of points with stabilizer $O(r)$ is

$$
r(n-d+r-1)-\left(\begin{array}{l}
r \\
2
\end{array}\right)=r(n-d)+\left(\begin{array}{l}
r \\
2
\end{array}\right) \leq n-d .
$$

Proposition 2.13. Suppose that $\Omega$ is equilibrium stress matrix so that $k>d+1$. Then $A(\Omega) / \operatorname{Eucl}(d)$ is a smooth stratified space with singularities of codimension at least 2. Furthermore, the singularities occur at classes of frameworks $[\sigma]$ where $\sigma$ lies in a proper affine subspace of $\mathbb{E}^{d}$.

Proof. Remember that $A(\Omega) \simeq K(\Omega)^{d}$ by Lemma 2.11. We can view this space as $K(\Omega) \otimes \mathbb{R}^{d}$ with $\operatorname{Eucl}(d)$ acting on the $d$-dimensional coordinates. We can turn this tensor product around: instead of thinking of $A(\Omega)$ as a $d$-tuple of points in $K(\Omega)$, think of it as a $k$-tuple of points in $\mathbb{E}^{d}$, with the diagonal action of $\operatorname{Eucl}(d)$. Concretely, you can always find $k$ vertices of the graph whose position determines the position of all other vertices of a framework inside $A(\Omega)$. Then $A(\Omega)$ is isomorphic to $\left(\mathbb{R}^{d}\right)^{k}$, thought of as the positions of these $k$ vertices.

Now by Lemma $2.12, A(\Omega) / \operatorname{Eucl}(d)$ is a smooth stratified space with singularities of codimension at least $k-d$, at frameworks which lie in a proper affine subspace. By assumption, $k>d+1$, and so this codimension is at least 2 .

2.3. The range. We now turn to defining the range.

Definition 2.14. Let $\Omega$ be an equilibrium stress matrix of some framework in $C^{d}(\Gamma)$. The space $B(\Omega)$ is defined to be $\ell(A(\Omega))$, the image in $\mathbb{R}^{e}$ of squared edge lengths of elements in $A(\Omega)$. Let $L(\Omega)$ to be the smallest linear subspace of $\mathbb{R}^{e}$ that contains $B(\Omega)$.

Note that $B(\Omega)$ is a semi-algebraic set. If the graph is generically locally rigid in $\mathbb{E}^{d}$ and $A(\Omega)$ contains a framework generic in $C^{d}(\Gamma)$, then $B(\Omega)$, like $A(\Omega) / \operatorname{Eucl}(d)$, is of dimension $k d-\left(\begin{array}{c}d+1 \\ 2\end{array}\right)$ (as the map $\ell$ is locally one-to-one from the quotient). The space $L(\Omega)$, for an appropriately chosen $\Omega$, will be the range space for our map. Our main task is to prove that 
for any generic $\rho$ and appropriately chosen $\Omega \in S(\rho), L(\Omega)$ is in fact the same dimension as $B(\Omega)$, or that $B(\Omega)$ is flat as in the following definition.

Definition 2.15. An irreducible semi-algebraic set $S$ in $\mathbb{R}^{n}$ is flat if it is contained in a linear subspace of $\mathbb{R}^{n}$ of the same dimension as $S$.

Note that this usage of flat is unrelated to the notion of flat families in algebraic geometry, and an algebraic variety is flat by this usage iff it is itself a linear space.

The key point in showing that $B(\Omega)$ is flat is Proposition 2.20, a standard theorem asserting that for any algebraic variety $V$, the contact locus of a generic hyperplane in the dual variety $V^{*}$ is flat. We recall these definitions and theorems. See [15] for a more complete treatment.

Definition 2.16. We say that a hyperplane $H$ is tangent to a homogeneous algebraic set $V \subset \mathbb{R}^{n}$ at a smooth point $x \in V$ if $T_{x}(V) \subset H$. For $\phi$ a functional in the dual space $\left(\mathbb{R}^{n}\right)^{*}$, we say that $\phi$ is tangent to $V$ at $x$ when $T_{x}(V) \subset \operatorname{ker}(\phi)$.

For a homogeneous algebraic set $V$ in $\mathbb{R}^{n}$, define the dual variety $V^{*} \subset\left(\mathbb{R}^{n}\right)^{*}$ to be the Zariski closure of the set of all functionals that are tangent to $V$ at a smooth point. The conormal bundle $C_{V} \subset \mathbb{R}^{n} \times\left(\mathbb{R}^{n}\right)^{*}$ is the Zariski closure of the pairs $(x, \phi)$ where $x$ is a smooth point of $V$ and $\phi$ is tangent to $V$ at $x$. There are two natural projections, $\pi_{1}: C_{V} \rightarrow V$ and $\pi_{2}: C_{V} \rightarrow V^{*}$. Above the smooth points of $V, C_{V}$ is smooth, although the projection onto $V^{*}$ need not be.

For $\phi \in V^{*}$, define the contact locus $V_{\phi}$ of $\phi$ to be $\pi_{1}\left(\pi_{2}^{-1}(\phi)\right)$. In particular, this contains the smooth points $x \in V$ so that $\phi$ is tangent to $V$ at $x$.

Remark 2.17. In Lemma 2.21 we relate the space of equilibrium stresses with the dual space to the measurement set $M$. In particular, this implies that the dual $\bar{M}^{*}$ is the Zariski closure of $S(\rho)$ as $\rho$ ranges over generic frameworks. In Proposition 2.23 we further identify $L(\Omega)$, for appropriate $\Omega$, as a contact locus.

Lemma 2.18. For $(x, \phi) \in C_{V}$ with $x$ smooth in $V$, we have that $\phi$ is tangent to $x$. (That is, the Zariski closure in the definition of $C_{V}$ does not add points above smooth points.) Similarly if $x \in V_{\phi}$ with $x$ smooth in $V$, $\phi$ is tangent to $x$. If $V$ is a homogeneous variety in $\mathbb{R}^{n}$, the conormal bundle $C_{V}$ always has dimension $n$ (independent of the dimension of $V$ ). If $V$ is irreducible, then so are $C_{V}$ and $V^{*}$.

Proof. See, e.g., [15, Section 2.1.4] for the proofs of these basic properties in the complex case. For the passage to the real case, let $V^{\mathbb{C}}$ be the complexification of $V$ and let $F: \mathbb{R}^{n} \rightarrow \mathbb{R}^{m}$ be an algebraic map that cuts out $V$, in the sense that $V$ is $F^{-1}(0)$ and $\operatorname{ker} d F_{x}=T_{x}(V)$ at all smooth points $x \in V$. Define

$$
\Gamma_{V}:=\left\{(x, \phi) \in V \times\left(\mathbb{R}^{n}\right)^{*} \mid \operatorname{rank}\left(\begin{array}{c}
\phi \\
d F_{x}
\end{array}\right) \leq n-\operatorname{dim} V\right\} .
$$

For any smooth point $x \in V$, the set of $\phi$ such that $(x, \phi)$ is in $\Gamma_{V}$ corresponds exactly to the set of functionals that are tangent to $V$ at $x$. Thus $C_{V}$ must be a union of irreducible components of $\Gamma_{V}$.

Most of the properties in the real case follow immediately from the observation that $\left(\Gamma_{V}\right)^{\mathbb{C}}=\Gamma_{V^{\mathbb{C}}}$, with the possible exception of irreducibility. For irreducibility of $C_{V}$, notice that from the properties of a complex conormal bundle, e.g., [15, Section 2.1.4], we know that $C_{V^{\mathbb{C}}}$ is an irreducible component of $\Gamma_{V^{\mathbb{C}}}$ but by, e.g., [29, Lemma 7], the components of $\left(\Gamma_{V}\right)^{\mathbb{C}}$ correspond to the components of $\Gamma_{V}$, so in particular there is one component containing all 
the tangents to smooth points. Irreducibility of $V^{*}$ follows from projection and the fact that from their definitions $V^{*}$ is the same as the Zariski closure of $\pi_{2}\left(C_{V}\right)$.

Proposition 2.19. For a homogeneous irreducible algebraic set $V$, the double dual $V^{* *}$ is $V$.

Proof idea. A short differential geometry argument shows that $C_{V}$ is the same as $C_{V^{*}}$ in an open neighborhood after permuting the factors. The equivalence of the entire bundles follows from irreducibility. It follows that $V^{* *}=V$. See [15, Section 2.1.5] for more.

Proposition 2.20. For a homogeneous irreducible algebraic set $V$ and a smooth point $\phi \in$ $V^{*}$, the contact locus $V_{\phi}$ is flat.

Proof idea. We wish to show that $\pi_{1}\left(\pi_{2}^{-1}(H)\right)$ is flat for smooth $\phi \in V^{*}$. Let us instead consider $\pi_{2}\left(\pi_{1}^{-1}(x)\right)$ for a smooth $x \in V$. By definition, this consists of all functionals vanishing on $T_{x}(V)$, which is a linear space. By the symmetry property of Proposition 2.19, we can apply this argument in the other direction to see that $\pi_{1}\left(\pi_{2}^{-1}(\phi)\right)$ is also a linear space. See [15, Section 2.1.6] for more.

Proposition 2.20 is sometimes called Bertini's Theorem; however, there are several theorems called Bertini's Theorem, some of which are quite different.

Note that the assumption that $\phi$ is smooth is crucial, and at singular points, the contact locus may have different structure. For example, consider the standard embedding in $\mathbb{R}^{3}$ of the 2 -torus (the surface of a donut sitting on a table). (In this case, since we are dealing with a non-homogeneous set, a tangent space is affine instead of linear.) At most points of the dual variety the contact locus is the single point on the torus with that specific affine tangent hyperplane. A single point is flat (in the affine sense). But for two exceptional hyperplanes (including the surface of the table) the contact locus is a circle, which is not flat. These two hyperplanes are non-smooth points of the dual variety.

We now turn to relating the above general construction to our setting of the measurement set $M$. Let $\bar{M}$ be the Zariski closure of $M . \bar{M}$ is homogeneous since $M$ is closed under multiplication by positive scalars. Let $C_{\bar{M}}, \bar{M}^{*}$, and $\bar{M}_{\omega}$ be the conormal, dual, and contact locus constructions applied to $\bar{M}$. (Here we think of $M$ as a subset of $\mathbb{R}^{e}$ and the equilibrium stress $\omega$ as an element of $\left.\left(\mathbb{R}^{e}\right)^{*}\right)$. We will translate freely between the stress $\omega$ and the corresponding stress matrix $\Omega$.

Lemma 2.21. Let $\rho \in C^{d}(\Gamma)$ be a framework so that $\ell(\rho)$ is smooth in $M$. Then if $\omega \in\left(\mathbb{R}^{e}\right)^{*}$ is tangent to $M$ at $\ell(\rho), \omega$ is an equilibrium stress for $\rho$. If furthermore $d \ell_{\rho}$ has maximal rank, then the converse holds: any equilibrium stress for $\rho$ is tangent to $M$ at $\ell(\rho)$.

Proof. Direct calculations show [2, p. 183, 10, Lemma 2.5, inter alia] that the space of equilibrium stress vectors at a point $\rho$, when thought of as a subspace of $\mathbb{R}^{e}$, constitute the annihilator of the span of $d \ell_{\rho}$ :

$$
S(\rho) \simeq \operatorname{ann}\left(\operatorname{span}\left(d \ell_{\rho}\right)\right)
$$

Finally, $\operatorname{span}\left(d \ell_{\rho}\right) \subset T_{\ell(\rho)}(M)$, with equality iff $d \ell_{\rho}$ has maximal rank. (It can happen that $\ell(\rho)$ is smooth even if $d \ell_{\rho}$ does not have maximal rank.) Thus $\operatorname{ann}\left(\operatorname{span}\left(d \ell_{\rho}\right)\right) \supset$ $\operatorname{ann}\left(T_{\ell(\rho)}(M)\right)$, again with equality if $d \ell_{\rho}$ has maximal rank. The result follows by definition of tangency. 
In particular, both directions of Lemma 2.21 apply at any generic framework $\rho$.

Motivated by Lemma 2.21, for $\omega \in \mathbb{R}^{e}$ let $B^{\circ}(\omega)$ be the "open contact locus": the set of smooth points $x \in M$ so that $\omega$ is tangent to $M$ at $x$.

Lemma 2.22. For $\rho$ a generic configuration and any $\omega \in S(\rho)$, the Euclidean closure of $B^{\circ}(\omega)$ is $B(\Omega)$.

Proof. By Lemma 2.21, if $\ell(\sigma) \in B^{\circ}(\omega), \sigma \in A(\Omega)$, so $B^{\circ}(\omega) \subset B(\Omega)$. Since $B(\Omega)$ is closed, we have one inclusion.

For the other direction, let $A^{g}(\Omega)$ be the points in $A(\Omega)$ which are generic in $C^{d}(\Gamma)$. The set $A^{g}(\Omega)$ contains $\rho$ by hypothesis and $A(\Omega)$ is a linear space and hence smooth. Thus by Lemma 2.9, $A^{g}(\Omega)$ is dense in $A(\Omega)$. Thus $\ell\left(A^{g}(\Omega)\right)$ is dense in $\ell(A(\Omega))$ which is equal to $B(\Omega)$. Clearly $B^{\circ}(\omega) \supset \ell\left(A^{g}(\Omega)\right)$. Thus the Euclidean closure of $B^{\circ}(\omega)$ contains the Euclidean closure of $\ell\left(A^{g}(\Omega)\right)$, which we just argued was $B(\Omega)$.

And now we are in a position to prove the flatness of our range space $B(\Omega)$.

Proposition 2.23. For $\rho$ a generic configuration and $\omega \in S(\rho)$ so that $\omega$ is generic in $\bar{M}^{*}$, the space $B(\Omega)$ is flat (i.e., $L(\Omega)$ is the same dimension as $B(\Omega)$ ).

Proof. From Lemma 2.4 $M$ is irreducible. By Proposition 2.20, the contact locus $\bar{M}_{\omega}$ is a linear space which we now identify with $L(\Omega)$. From Lemma $2.22, B^{\circ}(\omega)$ is dense in $B(\Omega)$ and thus $\operatorname{dim} B^{\circ}(\omega)=\operatorname{dim} B(\Omega) \leq \operatorname{dim} L(\Omega)$.

Again, due to Lemma 2.22, $L(\Omega)$ is also the smallest linear space containing $B^{\circ}(\omega)$. Since, by definition, $B^{\circ}(\omega)$ is contained in the linear space $\bar{M}_{\omega}$, we see that $L(\Omega) \subset \bar{M}_{\omega}$ and $\operatorname{dim} L(\Omega) \leq \operatorname{dim} \bar{M}_{\omega}$.

Now consider an open neighborhood $U$ of $\ell(\rho)$ in $\bar{M}$ that consists of smooth points that lie in $M$ (such a neighborhood must exist since $\ell(\rho)$ is generic). Then by Lemma $2.18, \bar{M}_{\omega} \cap U$ consists of points $x \in M$ where $\omega$ is tangent to $x$, i.e., $\bar{M}_{\omega} \cap U \subset B^{\circ}(\omega)$. But $\bar{M}_{\omega} \cap U$ is an open subset of the linear space $\bar{M}_{\omega}$, so $\operatorname{dim} \bar{M}_{\omega}=\operatorname{dim}\left(\bar{M}_{\omega} \cap U\right) \leq \operatorname{dim} B^{\circ}(\omega)$.

Since $\operatorname{dim} B(\Omega) \leq \operatorname{dim} L(\Omega), \operatorname{dim} L(\Omega) \leq \operatorname{dim} \bar{M}_{\omega}$, and $\operatorname{dim} \bar{M}_{\omega} \leq \operatorname{dim} B^{\circ}(\Omega)$ all the inequalities must be equalities, and in particular $\operatorname{dim} B(\Omega)=\operatorname{dim} L(\Omega)$ as desired.

Finally, we establish that for any generic framework $\rho$, we can find an $\omega \in S(\rho)$ which is generic in $\bar{M}^{*}$, so that we can apply Proposition 2.23 .

Lemma 2.24. If $\rho \in C^{d}(\Gamma)$ is generic, then there is an $\omega \in S(\rho)$ such that $(\ell(\rho), \omega)$ is generic in $C_{\bar{M}}$ and $\omega$ is generic inside of $\bar{M}^{*}$.

Proof. Consider the conormal bundle $C_{\bar{M}}$ with its two projections $\pi_{1}$ and $\pi_{2}$ to $\bar{M}$ and $\bar{M}^{*}$. Since $\ell(\rho)$ is generic in $\bar{M}$, it is smooth in $\bar{M}$. Thus, since $C_{\bar{M}}$ is smooth above smooth points of $\bar{M}$, there is a neighborhood $N$ of $\ell(\rho)$ so that $\pi_{1}^{-1}(N)$ is a smooth semi-algebraic set. Applying Lemma 2.8 to the restriction of $\pi_{1}$ to $\pi_{1}^{-1}(N)$ guarantees a point $(\ell(\rho), \omega) \in$ $\pi_{1}^{-1}(\ell(\rho))$ that is generic in $\pi_{1}^{-1}(N)$ and thus also in $C_{\bar{M}}$. By Lemma 2.7, $\omega \in \bar{M}^{*}$ is also generic. This $\omega$ is an equilibrium stress for $\rho$ by Lemmas 2.18 and 2.21 .

2.4. Identifying the contact locus. We now digress briefly to give a somewhat more explicit description of the space $L(\Omega)$. This is not necessary for any of our proofs, but may aid in understanding. 
Definition 2.25. For a set $S \subset \mathbb{E}^{n}$ and $d \in \mathbb{N}$, let $\operatorname{chord}^{d}(S)$ be the $d^{\prime}$ th chord set of $S$, the union of all simplices with $d$ vertices, all in $S$. For instance, when $d=2$ we add chords connecting all pairs of points in $S$. (For a variety $V$, the Zariski closure of $\operatorname{chord}^{2}(V)$ is the secant variety $\sec (V)$, where we add complete lines instead of segments.)

Lemma 2.26. The space $B(\Omega)$ is $\operatorname{chord}^{d}(\ell(K(\Omega)))$.

Proof. The squared edge lengths of a framework $\rho$ are computed by summing the squared edge lengths of each coordinate projection of $\rho$. Since $A(\Omega)$ is $K(\Omega)^{d}, B(\Omega)$ is the $d$-fold Minkowski sum of $\ell(K(\Omega))$ with itself. Because $\ell(K(\Omega))$ is invariant under scaling by positive reals, this Minkowski sum coincides with $\operatorname{chord}^{d}(\ell(K(\Omega)))$.

Definition 2.27. For $a, b \in C^{1}(\Gamma)$, define a "dot product" $\langle a, b\rangle \in \mathbb{R}^{e}$ by

$$
\langle a, b\rangle(\{w, u\}):=(a(w)-a(u)) \cdot(b(w)-b(u))
$$

for each edge $\{u, w\} \in \mathcal{E}$. Define the dot product space $D(\Omega)$ to be the linear span of $\langle a, b\rangle$ for all $a, b \in K(\Omega)$.

Lemma 2.28. The space $L(\Omega)$ is the same as $D(\Omega)$.

Proof. The image $\ell(K(\Omega))$ is contained in $D(\Omega)$, as it is just the space of all $\langle a, a\rangle$ for $a \in K(\Omega)$. Moreover, the linear span of $\ell(K(\Omega))$ is in fact equal to $D(\Omega)$, as $\langle a, b\rangle=$ $\frac{1}{2}(\langle a+b, a+b\rangle-\langle a, a\rangle-\langle b, b\rangle)$. From Lemma 2.26, the linear span of $\ell(K(\Omega))$ is the same as the linear span of $B(\Omega)$, which is $L(\Omega)$ by definition.

A priori, if $K(\Omega)$ is $k$-dimensional, the dimension of $D(\Omega)$ could be as big as $\left(\begin{array}{l}k \\ 2\end{array}\right)$. (There are $\left(\begin{array}{c}k+1 \\ 2\end{array}\right)$ dot products between vectors forming a basis of $K(\Omega)$. However, dot products with the vector of all ones vanish because of the subtraction in the definition of $\langle a, b\rangle$.) On the other hand, the dimension of $B(\Omega)$ is only $k d-\left(\begin{array}{c}d+1 \\ 2\end{array}\right)$. When $\Gamma$ has a minimal stress kernel realized by $\Omega, k=d+1$ and $\operatorname{dim}(B(\Omega))$ agrees with this estimate for $\operatorname{dim}(D(\Omega))$. The crux of Proposition 2.23 is that in fact, even when $\Gamma$ does not have minimal stress kernel, if $\Omega$ is generic in $\bar{M}^{*}$ then $B(\Omega)$ is a flat space, and thus $D(\Omega)$ is in fact only of dimension $k d-\left(\begin{array}{c}d+1 \\ 2\end{array}\right)$. This means that there must be some linear dependence between the dot products defining $D(\Omega)$.

2.5. The map. We now turn to our main map.

Definition 2.29. Let $\Omega$ be an equilibrium stress matrix of some framework in $C^{d}(\Gamma)$. The map $\bar{\ell}$ is the restriction of $\ell$ to a map between the spaces $A(\Omega) / \operatorname{Eucl}(d)$ and $L(\Omega)$.

In this section, we show that, for any generic framework $\rho$ and stress $\Omega \in S(\rho)$ so that $\Omega$ is generic in $\bar{M}^{*}$, the map $\bar{\ell}$ has a well-defined mod-two degree which is 0 , and then deduce the theorem. (Recall that the existence of such a generic $\Omega$ is guaranteed by Lemma 2.24.)

First we recall a few standard elementary properties of regular values, proper maps, and degrees. Because our maps are not always locally injective or surjective, we use a slightly generalized definition of regular value and version of Sard's theorem.

Definition 2.30. Let $f: X \rightarrow Y$ be a smooth map between smooth manifolds $X$ and $Y$. Let $r$ be the maximal rank of the linearization $d f_{x}$ of $f$ for any $x \in X$. We say $x \in X$ is a regular point if $d f_{x}$ has rank $r$. We say $y \in Y$ is a regular value if every $x \in f^{-1}(y)$ is a regular point. Otherwise, $y$ is called a critical value. 
Theorem 2.31 (Sard [25]). Let $f: X \rightarrow Y$ be a smooth map between smooth manifolds, and let $r$ be the maximal rank of $d f_{x}$ for $x \in X$. The critical values of $f$ have $r$-dimensional measure 0 .

Proposition 2.32. Let $X$ and $Y$ be semi-algebraic sets that are manifolds and $f$ be a polynomial map with maximal rank $r$, all defined over the field $\mathbf{k}$. Then the critical values form a semi-algebraic subset of $Y$, defined over $\mathbf{k}$, of dimension less than $r$. In particular, all generic points of $f(X)$ are regular values.

Proof. See, e.g., [5, Theorem 9.6.2].

Definition 2.33. A proper map $f: X \rightarrow Y$ between topological spaces is a continuous map so that the inverse image of a compact set is compact.

Examples of proper maps include the identity and any map from a compact space. For our purposes, we will need the following:

Lemma 2.34. The length measurement map associated to any connected graph, once we quotient the domain by the group $\operatorname{Trans}(d)$ of translations, is proper, as is its restriction to $A(\Omega)$ for any equilibrium stress matrix $\Omega$. That is, the maps

$$
\begin{aligned}
& \ell: C^{d}(\Gamma) / \operatorname{Trans}(d) \rightarrow \mathbb{R}^{e} \\
& \ell: A(\Omega) / \operatorname{Trans}(d) \rightarrow \mathbb{R}^{e}
\end{aligned}
$$

are both proper.

Proof. A compact subset $P \subset \mathbb{R}^{e}$ is bounded, so gives a bound on the edge lengths. This in turn gives a bound on how far any vertex in $\Gamma$ can be from some fixed base vertex, so $\ell^{-1}(P)$ is bounded in $C^{d}(\Gamma) / \operatorname{Trans}(d)$ or the subspace $A(\Omega) / \operatorname{Trans}(d)$. Since $\ell^{-1}(P)$ is also closed, it is compact.

There is a notion of degree of proper maps between manifolds of the same dimension. The following theorem is standard, and is typically proved using homology [28, Theorem 8.12, inter alia].

Theorem 2.35. If $X$ and $Y$ are manifolds of the same dimension, with $Y$ connected, and $f: X \rightarrow Y$ is a proper map, then there is an element $\operatorname{deg} f$ in $\mathbb{Z} / 2$, invariant under proper isotopies of $f$. If $X, Y$, and $f$ are all smooth, then the degree is equal to the number of preimages of any regular value, taken modulo 2.

We will want to compute the degree in a case when $X$ is not quite a manifold, but rather has singularities of codimension 2. We use the following version.

Corollary 2.36. If $X$ is a smooth stratified space with singularities of codimension at least 2, $Y$ is a smooth, connected manifold of the same dimension as $X$, and $f: X \rightarrow Y$ is a proper, smooth map, then there is an element $\operatorname{deg} f$ in $\mathbb{Z} / 2$, invariant under proper isotopies of $f$. The degree is equal to the number of preimages of any regular value in $Y$, taken modulo 2.

Here by a "regular value" we mean a point in $Y$ so that every preimage is a smooth, regular point in $X$. The condition that the singularities have codimension at least 2 is crucial; otherwise, for instance, the inclusion of the interval $[0,1]$ in $\mathbb{R}$ would qualify, and the degree is obviously not invariant there. 
Proof. Let $X^{\text {sing }}$ be the set of singular points of $X$. Let $Y^{\prime}=Y \backslash f\left(X^{\text {sing }}\right)$, and let $X^{\prime}=$ $f^{-1}\left(Y^{\prime}\right)$. Then $X^{\prime}$ and $Y^{\prime}$ are both smooth manifolds by construction. By Lemma 2.37 below, the restriction of $f$ to a function from $X^{\prime}$ to $Y^{\prime}$ is still proper. The smooth image of the stratified space $X^{\text {sing }}$ is itself a stratified set, with dimension no bigger: $\operatorname{dim}\left(f\left(X^{\operatorname{sing}}\right)\right)<$ $\operatorname{dim}\left(X^{\operatorname{sing}}\right)$. Therefore $Y^{\prime}$ is still connected, as we have removed a subset of codimension at least 2 from $Y$. We can therefore apply Theorem 2.35 to find $\operatorname{deg} f$ as the degree of the restriction from $X^{\prime}$ to $Y^{\prime}$.

Lemma 2.37 (Excision). If $f: X \rightarrow Y$ is a proper map and $Y^{\prime} \subset Y$ is an arbitrary subset, let $X^{\prime}=f^{-1}\left(Y^{\prime}\right)$. Then the restriction of $f$ to $X^{\prime}, f^{\prime}: X^{\prime} \rightarrow Y^{\prime}$, is proper.

Proof. For any compact subset $P$ of $Y^{\prime}, P$ is also compact as a subset of $Y$. Since $f^{\prime-1}(P)$ is the same as $f^{-1}(P)$, it is compact.

More generally, there is an integer-valued degree for maps between oriented manifolds. Our domain space is not in general oriented, so we only have a mod-two degree, but that is enough for us.

Applying these results to our map $\bar{\ell}$, we see that:

Lemma 2.38. Suppose that $\Gamma$, a graph with $d+2$ or more vertices, is generically locally rigid in $\mathbb{E}^{d}$ and does not have minimal stress kernel in $\mathbb{E}^{d}, \rho$ is a generic framework in $C^{d}(\Gamma)$, and $\omega \in S(\rho)$ is generic in $\bar{M}^{*}$. Then the resulting map $\bar{\ell}$ from $A(\Omega) / \operatorname{Eucl}(d)$ to $L(\Omega)$ has a mod-two degree of 0 .

Proof. By Lemma 2.34 the map $\bar{\ell}$ is proper. (Local rigidity implies that $\Gamma$ is connected.)

Since $\Gamma$ does not have a minimal stress kernel and $\rho$ is generic, $\operatorname{dim}(K(\Omega))>d+1$ for all $\Omega \in S(\rho)$. Thus by Proposition 2.13, the domain is a smooth stratified space with singularities of codimension 2 or greater. By Proposition 2.23, $L(\Omega)$ (the range of $\bar{\ell}$ ) has the same dimension as $B(\Omega)$, which is the same dimension as the domain by local rigidity. So by Corollary 2.36 it has a well defined degree mod-two.

Since all squared edge lengths in the image of $\ell$ are positive, any point in $L(\Omega)$ with some negative edge lengths has no preimage in $A(\Omega) / \operatorname{Eucl}(d)$ and is automatically regular. Hence the mod-two degree must be 0 .

For Lemma 2.38 to be useful, we must see that $\ell(\rho)$ is a regular value of $\bar{\ell}$.

Lemma 2.39. Suppose that $\Gamma$, a graph with $d+2$ or more vertices, is generically locally rigid in $\mathbb{E}^{d}$, and $\rho$ is a generic framework in $C^{d}(\Gamma)$. Then $\ell(\rho)$ is a regular value of both $\ell$ and $\bar{\ell}$.

Proof. Since $\ell(\rho)$ is a generic point of $M$, by Sard's Theorem $\ell(\rho)$ is a regular value of $\ell$, proving the first part of the statement. At each preimage $\sigma$ of a regular value, $d \ell_{\sigma}$ is of maximal rank, and so $\sigma$ is infinitesimally rigid. In particular, $\sigma$ must have a $d$-dimensional affine span, and hence cannot have a non-trivial stabilizer in $\operatorname{Eucl}(d)$. Then by Proposition 2.13 every point in $\bar{\ell}^{-1}(\ell(\rho))$ is a smooth point in $A(\Omega) / \operatorname{Eucl}(d)$.

Since $d \ell_{\sigma}$ is of maximal rank, it is injective from the tangent of $C^{d}(\Gamma) / \operatorname{Eucl}(d)$ to $\mathbb{R}^{e}$. The map $d \bar{\ell}_{\sigma}$ is the restriction of $d \ell_{\sigma}$ from the tangent space to $C^{d}(\Gamma) / \operatorname{Eucl}(d)$ to a subspace. Restriction preserves injectivity of the linearization of a smooth map, so $\ell(\rho)$ is also a regular value for $\bar{\ell}$.

The regularity of $\ell(\rho)$ with respect to $\bar{\ell}$ can also be proved by using Lemma 6.2 .

And now we are in position to complete the proof of our main Theorem. 

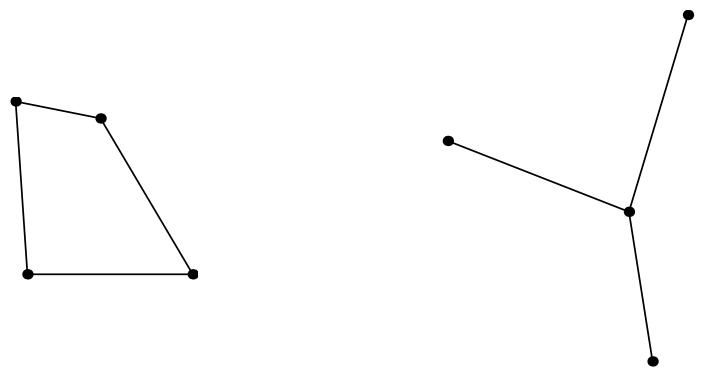

Figure 2. Generic locally flexible frameworks in $\mathbb{E}^{2}$

Proof of Theorem 1.14. By Lemma 2.24, for any generic $\rho$ there is an $\omega \in S(\rho)$ that is generic in $\bar{M}^{*}$. Choose the associated equilibrium stress matrix $\Omega$ to define $\bar{\ell}$. From Lemma 2.38, the mod-two degree of $\bar{\ell}$ is 0 . From Lemma 2.39, $\ell(\rho)$ is a regular value of $\bar{\ell}$. Thus there must be an even number of points in $\bar{\ell}^{-1}(\ell(\rho))$. Since there is at least one point in the preimage, namely $[\rho]$ itself, there must be another point in $A(\Omega) / \operatorname{Eucl}(d)$, and thus an incongruent framework in $C^{d}(\Gamma)$ that has the same edge lengths as $\rho$.

\section{EXAMPLES}

Here we give a few examples of how the various spaces constructed in Section 2 work out in practice. We consider both cases where Theorem 1.14 applies to show that generic frameworks are not globally rigid, and cases where it does not apply.

Throughout this section, $\rho$ is a generic framework for whichever graph we are looking at and $\omega \in S(\rho)$ is chosen to be generic in $\bar{M}^{*}$. (In particular, $\operatorname{dim}(K(\Omega))=k_{\min }$.)

3.1. Locally flexible graphs. The first case we consider is that of graphs that are generically locally flexible in $\mathbb{E}^{d}$, as in Figure 2.

The space $A(\Omega)$ contains all affine transforms of any flex of $\rho$, and possibly more. This can be seen by using the main argument from [10]: any flex $\sigma$ of $\rho$ maps to $\ell(\rho)$ in $M$, which is smooth since $\rho$ is generic. We therefore have $\operatorname{span}\left(d \ell_{\sigma}\right) \subset T_{\ell(\rho)} M=\operatorname{span}\left(d \ell_{\rho}\right)$. In particular, any equilibrium stress for $\rho$ is also an equilibrium stress for $\sigma$, so $\sigma \in A(\Omega)$.

A simple example of such a graph is the tripod graph in $\mathbb{E}^{2}$ as on the right in Figure 2. In this case, the only equilibrium stress vector is the zero stress, and $A(\Omega)$ contains all embeddings of $\Gamma$. Note that in this case, $L(\Omega)$ is three dimensional, which is the same size as one would find for a generically globally rigid graph.

3.2. Not redundantly rigid. Here we suppose that $\rho$ is generically locally rigid, but becomes not generically locally rigid when some edge $\{i, j\}$ is removed from $\Gamma$, as shown for example in Figure 3. In other words, we suppose that the graph can be flexed, leaving all edge lengths unchanged except for edge $\{i, j\}$, which is changed. These graphs are not generically globally rigid by a result of Hendrickson [17]; we will see how this works in our setting. For a generic framework $\rho$, we can find, in the span of $d \ell_{\rho}$, a vector with all zeros except for the entry associated with edge $\{i, j\}$ (since by definition there is a flex changing this edge length and no others). Since the equilibrium stress vectors annihilate $\operatorname{span}\left(d \ell_{\rho}\right)$, we conclude that any equilibrium stress $\Omega$ has a zero at entry $\{i, j\}$. (In fact, a locally rigid generic framework is redundantly rigid at edge $\{i, j\}$ iff it has an equilibrium stress with a non-zero entry at that edge.) 

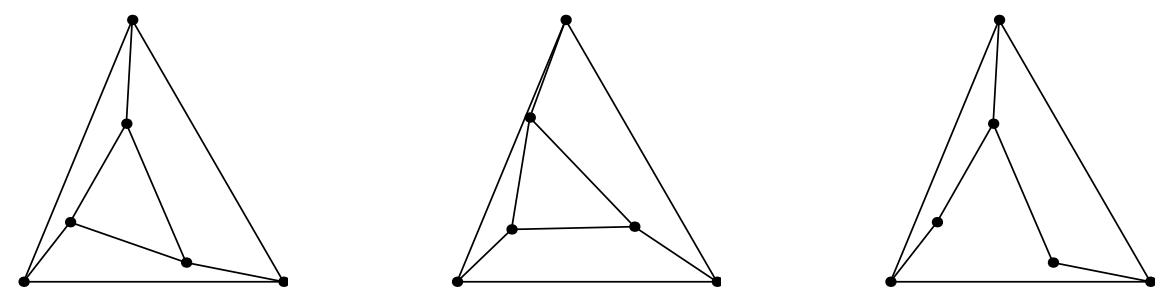

FiguRE 3. A framework that is not redundantly rigid in $\mathbb{E}^{2}$, with graph given by a triangular prism. From left to right, we have a framework $\rho$, the global flex guaranteed by the theorem, and the locally flexible framework $\rho^{\prime}$ obtained by deleting a suitable edge.
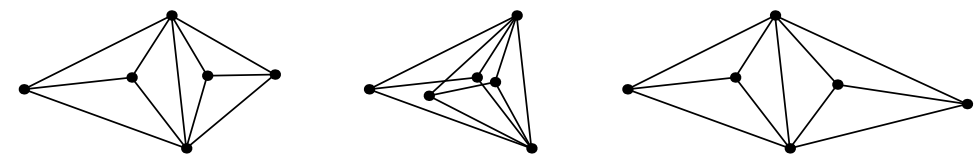

Figure 4. From left to right, a framework $\rho$ that is not 3-connected in $\mathbb{E}^{2}$ (and therefore not globally rigid), its global flex, and another framework in $A(\Omega)$.

Define $\Gamma^{\prime}$ to be the graph obtained by deleting this edge, and $\rho^{\prime}$ to be the resulting framework. Since every equilibrium stress for generic frameworks has zeroes at entry $\{i, j\}$, the equilibrium stresses for $\Gamma$ and $\Gamma^{\prime}$ are the same; that is, $\overline{M(\Gamma)}^{*}={\overline{M\left(\Gamma^{\prime}\right)}}^{*}$. In particular, we may think of our chosen $\Omega$ as an equilibrium stress for $\rho^{\prime}$, and it is then generic in ${\overline{M\left(\Gamma^{\prime}\right)}}^{*}$. By the analysis of generically locally flexible frameworks in Section 3.1, $A(\Omega)$ contains all affine transforms of flexes of $\rho^{\prime}$. In terms of $\rho$, we would say that $A(\Omega)$ contains all affine transforms of frameworks of $\Gamma$ that have the same edge lengths as $\rho$ except on the edge $\{i, j\}$.

It is also instructive to picture the relationship between the measurement set $M(\Gamma)$ in $\mathbb{R}^{e}$ and the measurement set $M\left(\Gamma^{\prime}\right)$ in $\mathbb{R}^{e-1}$. Because the framework is not redundantly rigid, $M(\Gamma)$ projects onto $M\left(\Gamma^{\prime}\right)$ (by forgetting one coordinate in $\mathbb{R}^{e}$ ), and the fiber over a generic point $\ell\left(\rho^{\prime}\right)$ contains an interval. Accordingly, $L(\Omega) \subset \mathbb{R}^{e}$ for $\Gamma$ is the direct sum of the corresponding $L(\Omega) \subset \mathbb{R}^{e-1}$ for $\Gamma^{\prime}$ and the vector in the direction of the missing edge.

3.3. Graphs that are not $(d+1)$-connected. A graph is not $(d+1)$-connected if there is a set of at most $d$ vertices whose removal leaves a disconnected graph. These graphs are not generically globally rigid in $\mathbb{E}^{d}[17]$. If the separating set has $d$ vertices, they span an "interface" hyperplane whose removal splits the graph into two halves, and we can reflect one half across the interface. If the separating set has fewer vertices, the graph is not even generically locally rigid, as we can pivot the two halves around the interface.

Let $\Gamma^{\prime}$ be one of the two halves of the graph, together with the vertices and edges in the separating set, and let $\rho^{\prime}$ be the corresponding framework. Any equilibrium stress $\Omega$ in $S(\rho)$ induces a non-equilibrium stress on $\rho^{\prime}$. The resulting forces on the vertices of $\Gamma^{\prime}$ must have no net translational and rotational effect (as is true for any stress, equilibrium or not). On the other hand, these forces are non-zero only on the $d$ vertices of the interface. It is then easy to see that the forces must lie entirely within the plane of the interface. 


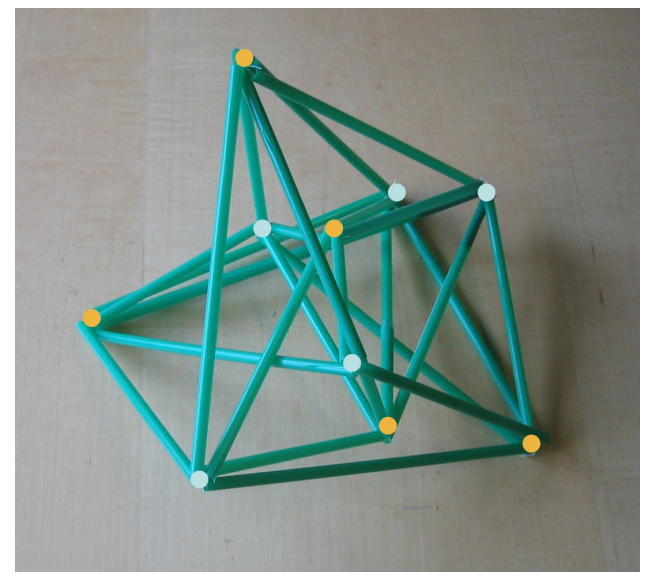

Figure 5. A framework of $K_{5,5}$ in 3 dimensions

Therefore the forces on the interface vertices are unchanged by affinely squashing one half towards the interface while leaving the other half alone. Thus $A(\Omega)$ includes frameworks where one applies two different affine transforms to the two halves, with the constraint that they agree on the interface. In particular, this includes the global flex that entirely folds one side across the interface.

3.4. Bipartite graphs. We next consider certain complete bipartite frameworks which (as Connelly showed) satisfy Hendrickson's conditions but are still not generically globally rigid [9]. The first example is $K_{5,5}$ in $\mathbb{E}^{3}$, as in Figure 5, which we focus on first. Let the vertices be $v_{1}, \ldots, v_{10}$ so that the each of $v_{1}, \ldots, v_{5}$ is connected to each of $v_{6}, \ldots, v_{10}, 25$ edges in all. We have that $C^{3}\left(K_{5,5}\right)$ is 30-dimensional. Since this graph is generically locally rigid, the measurement set $M$ has dimension $30-\operatorname{dim}(\operatorname{Eucl}(3))=24$ inside $\mathbb{R}^{25}$. We therefore have a 1-dimensional space $S(\rho)$ of equilibrium stress vectors at a generic framework, which we now identify, following Bolker and Roth [6]. (In fact, Bolker and Roth went the other way: they used the identification of the space of equilibrium stresses to identify which bipartite graphs are generically locally rigid, including $K_{5,5}$ in $\mathbb{E}^{3}$.) We will write $v_{1}, \ldots, v_{10}$ for the 10 points in $\mathbb{E}^{3}$ at the generic framework $\rho$.

The 5 points $v_{1}, \ldots, v_{5}$ in $\mathbb{E}^{3}$ satisfy an affine relation

$$
\begin{array}{r}
a_{1} v_{1}+\cdots+a_{5} v_{5}=0 \\
a_{1}+\cdots+a_{5}=0
\end{array}
$$

where not all $a_{i}$ are 0 . Since the $v_{i}$ are generic, this relation is unique up to scale. Similarly there is a unique up to scale affine relation between $v_{6}, \ldots, v_{10}$ :

$$
\begin{array}{r}
b_{6} v_{6}+\cdots+b_{10} v_{10}=0 \\
b_{6}+\cdots+b_{10}=0 .
\end{array}
$$

Now form vectors $\vec{a}=\left(a_{1}, \ldots, a_{5}\right)$ and $\vec{b}=\left(b_{6}, \ldots, b_{10}\right)$ and consider the block matrix

$$
\Omega=\left(\begin{array}{cc}
0 & \vec{a}^{T} \vec{b} \\
\vec{b}^{T} \vec{a} & 0
\end{array}\right) .
$$


(That is, the $(i, j)$ entry of $\Omega$ is $a_{i} b_{j}$ or $a_{j} b_{i}$ if either of these is defined, and 0 otherwise.) Then it follows that $\Omega$ is an equilibrium stress matrix and so spans the space $S(\rho)$.

In particular, since $\Omega$ visibly has $\operatorname{rank} 2, \operatorname{dim} K(\Omega)=8$. The basis vectors are

(1) a vector of 3 coordinate projections of $v_{1}$ through $v_{5}$ and five 0 's in the last 5 positions,

(2) a vector of five 1's and five 0's, and

(3) five 0 's in the first 5 positions followed by the 3 coordinate projections of $v_{6}$ through $v_{10}$

(4) a vector of five 0's and five 1's.

The space $A(\Omega)$ is also easy to identify: it is the 24-dimensional space of configurations $w_{1}, \ldots, w_{10}$, where $w_{1}, \ldots, w_{5}$ are an affine transform of $v_{1}, \ldots, v_{5}$ and $w_{6}, \ldots, w_{10}$ are an affine transform of $v_{6}, \ldots, v_{10}$, but the two affine transforms need not be the same. This guarantees that $w_{1}, \ldots, w_{5}$ satisfy the affine relation given by $\vec{a}$ and $w_{6}, \ldots, w_{10}$ satisfy the affine relation given by $\vec{b}$, and so these configurations satisfy the equilibrium stress matrix $\Omega$.

It follows that $L(\Omega)$ is an 18-dimensional space. We can think of it as the space $D(\Omega)$ as in Definition 2.27.

More generally, Bolker and Roth showed that a generic framework of $K_{n, m}$ in $\mathbb{E}^{d}$ with $n, m \geq d+2$ and $n+m=\left(\begin{array}{c}d+2 \\ 2\end{array}\right)$ is locally rigid and has equilibrium stresses only of the form

$$
\Omega=\left(\begin{array}{cc}
0 & X \\
X^{T} & 0
\end{array}\right)
$$

where $X$ is a $n \times m$ matrix in which each column is an affine linear relation among $v_{1}, \ldots, v_{n}$ and each row is an affine linear relation among $v_{n+1}, \ldots, v_{n+m}$. In particular, the framework is redundantly rigid (since there are equilibrium stresses with non-zero entries one every edge). The rank of $X$ is at $\operatorname{most} \min (n, m)-d-1$, the rank of $\Omega$ is twice as large, and

$$
k_{\min }=n+m-\operatorname{rank}(\Omega)=|n-m|+2(d+1)>d+1
$$

so (as Connelly stated) these graphs are not generically globally rigid.

3.5. Generically globally rigid graphs. Finally we consider a generic framework $\rho$ in $\mathbb{E}^{d}$ that does satisfy Connelly's condition, so Theorem 1.14 does not apply and the framework is generically globally rigid. It is instructive to see where the proof breaks. In this case, for every $\Omega \in S(\rho)$ that is generic in $\bar{M}^{*}, K(\Omega)$ is only $(d+1)$-dimensional. The space $A(\Omega)$ is then just $\operatorname{Aff}(\rho)$, the space of affine transforms of $\rho$.

Let $\rho_{i}$ be the projection of $\rho$ onto the $i$ th coordinate. Then by Lemma 2.28, $L(\Omega)$ is spanned by the $\left(\begin{array}{c}d+1 \\ 2\end{array}\right)$ different vectors $\left\langle\rho_{i}, \rho_{j}\right\rangle$. These are all linearly independent, since, as shown in [10, Proposition 4.3], whenever Connelly's condition is satisfied, the edges of $\rho$ do not lie on a conic at infinity. (A conic at infinity is, in our language, a linear dependence among the $\left\langle\rho_{i}, \rho_{j}\right\rangle$.)

Moreover, by [10, Proposition 4.2], whenever the edges of $\rho$ do not lie on a conic at infinity, $\ell$ is injective on $\operatorname{Aff}(\rho) / \operatorname{Eucl}(d)$. Thus in our case, $B(\Omega)$ is homeomorphic to $A(\Omega) / \operatorname{Eucl}(d)$. In particular, $B(\Omega)$ has a boundary (as it is a proper subset of $L(\Omega)$ ) and so must $A(\Omega) / \operatorname{Eucl}(d)$. This boundary may be thought of as a singularity of codimension 1 in the domain, and thus our degree argument does not apply.

More explicitly, $\ell(\operatorname{Aff}(\rho))$ is equivalent to a semidefinite cone, as we now explain. Let $\operatorname{span}(\ell(\operatorname{Aff}(\rho)))$ be the linear span of the image space $\ell(\operatorname{Aff}(\rho))$. Use the basis $\left\langle\rho_{i}, \rho_{j}\right\rangle$ to define an isomorphism $\phi$ from $\operatorname{span}(\ell(\operatorname{Aff}(\rho)))$ to the space of symmetric $d \times d$ matrices, 
sending $\left\langle\rho_{i}, \rho_{j}\right\rangle$ to $e_{i j}$, where

$$
e_{i j}= \begin{cases}\text { matrix with a } 1 \text { at position }(i, i) \text { and } 0 \text { 's elsewhere } & i=j \\ \text { matrix with a } 1 / 2 \text { at positions }(i, j) \text { and }(j, i) \text { and } 0 \text { 's elsewhere } & i \neq j .\end{cases}
$$

Lemma 3.1. For a framework $\rho$ whose edges do not lie on a conic at infinity and $\phi$ defined as above, $\phi(\ell(\operatorname{Aff}(\rho)))$ is the set of positive semidefinite matrices.

Proof. Since $\ell$ is invariant under translation of $\rho$, it suffices to consider $\phi(\ell(U \rho))$, where $U=\left(u_{j}^{i}\right)_{i, j=1}^{d}$ is a linear map (rather than a general affine map). We then find

$$
\begin{aligned}
\phi(\ell(U(\rho))) & =\phi\left(\sum_{j}\left\langle\sum_{i} u_{j}^{i} \rho_{i}, \sum_{i} u_{j}^{i} \rho_{i}\right\rangle\right) \\
& =\sum_{j, i, i^{\prime}} u_{j}^{i} u_{j}^{i^{\prime}} e_{i i^{\prime}} \\
& =U^{T} U .
\end{aligned}
$$

The $d \times d$ positive semidefinite matrices are exactly those matrices that can be written as $U^{T} U$ for a $d \times d$ matrix $U$.

In particular, when the graph has minimal stress kernel and $\rho$ and $\Omega$ are generic, $A(\Omega)=$ $\operatorname{Aff}(\rho)$, and thus $B(\Omega)$ and $A(\Omega) / \operatorname{Eucl}(d)$ are isomorphic to the semidefinite cone in $\phi(L(\Omega))$. The number of preimages in $A(\Omega) / \operatorname{Eucl}(d)$ of a point in $L(\Omega)$ is 1 for points inside the positive semidefinite cone. (At non-generic points, there may be more preimages in $C^{d}(\Gamma) / \operatorname{Eucl}(d)$ that are not in $A(\Omega)$ ). The number of preimages is 0 for points outside this cone (such as points in $\mathbb{R}^{e}$ with any negative coordinate). Thus the degree is definitely not independent of the generic target point we consider.

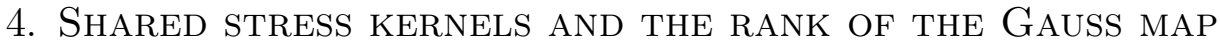

In this section we give an alternate characterization of global rigidity, in terms of the shared stress kernel.

Definition 4.1. The shared stress kernel of a framework $\rho$ is the intersection of all stress kernels: $K(\rho):=\bigcap_{\Omega \in S(\rho)} K(\Omega)$. It is isomorphic to the space of frameworks of $\Gamma$ in $\mathbb{E}^{1}$ which satisfy all the equilibrium stress matrices in $S(\rho)$ (and maybe more). Let the shared stress nullity $k_{\mathrm{sh}}(\Gamma, d)$ be $\operatorname{dim}(K(\rho))$ for a generic framework $\rho$.

Remark 4.2. As for $k_{\min }$, the shared stress nullity $k_{\mathrm{sh}}$ is independent of the generic framework $\rho$. One way to see this is to note that the intersection in the definition of $k_{\text {sh }}$ is achieved by considering a sufficiently large finite number of equilibrium stress matrices $\Omega_{i} \in S(\rho)$. The intersection of these kernels is also the kernel of a larger matrix $\Omega^{\prime}=\left(\Omega_{1} \Omega_{2} \cdots \Omega_{n}\right)$ obtained by concatenating the $\Omega_{i}$. But $\Omega^{\prime}$ can be constructed by algebraic functions (as in the proof of Lemma 5.8) so its rank is maximal at generic points by Lemma 5.6.

Alternately, we can use the equivalence with the Gauss rank in Proposition 4.6 below to show the same fact.

Remark 4.3. By definition, $k_{\min } \geq k_{\mathrm{sh}}$. It frequently happens that $k_{\min }=k_{\mathrm{sh}}$, but it is not true in general that the analogous numbers are the same. For instance, the matrices

$$
\left(\begin{array}{lll}
0 & a & b \\
a & 0 & 0 \\
b & 0 & 0
\end{array}\right)
$$


for $a, b \in \mathbb{R}$ all have rank 2 (and so a 1-dimensional kernel); but the intersection of their kernels is zero. A similar example occurs in practice for bipartite graphs; for frameworks of $K_{n, m}$ in $\mathbb{E}^{d}$ with $n, m \geq d+2, n+m=\left(\begin{array}{c}d+2 \\ 2\end{array}\right)$, and $n \neq m$, it follows from the description of the equilibrium stresses recalled in Section 3.4 that

$$
\begin{aligned}
k_{\min } & =|n-m|+2(d+1) \\
k_{\mathrm{sh}} & =2(d+1)<k_{\min } .
\end{aligned}
$$

The second equation follows from the fact that the only frameworks that satisfy all of the stresses of $\rho$ must be related to $\rho$ by an affine transform on each of the two sides of the bipartite graph. (For instance, $K_{7,8}$ in $\mathbb{E}^{4}$ satisfies these constraints, and has $k_{\min }=11$ and $\left.k_{\mathrm{sh}}=10.\right)$

Although $k_{\min }$ and $k_{\mathrm{sh}}$ are different in general, either one can be used in a test for global rigidity, so if $k_{\min }=v-d-1$, then $k_{\mathrm{sh}}=v-d-1$ as well:

Theorem 4.4. A graph $\Gamma$ with $d+2$ or more vertices is generically globally rigid in $\mathbb{E}^{d}$ if and only if $k_{s h}(\Gamma, d)=d+1$.

One direction of Theorem 4.4 is a corollary of Theorem 1.14, as $k_{\mathrm{sh}} \leq k_{\mathrm{min}}$. For the other direction, we strengthen Connelly's argument, using the same proof.

4.1. The Gauss map and its rank. Before continuing we point out that there is an alternate way of interpreting the shared stress kernel, by using the rank of maps.

Definition 4.5. The rank of an algebraic or rational map between irreducible algebraic varieties is rank of its linearization at generic points in the domain. (This is also the maximum rank of its linearization.) Equivalently, it is the dimension of the image semi-algebraic set.

Thus Theorem 1.6 says that a graph is generically locally rigid in $\mathbb{E}^{d}$ if and only if the rank of the map $\ell$ is "the largest it can be".

Now assume that $\Gamma$ is generically locally rigid in $\mathbb{E}^{d}$, and let $t=v d-\left(\begin{array}{c}d+1 \\ 2\end{array}\right)$ be the dimension of the measurement set $M$. Consider now the Gauss map, the map that takes each smooth point of $M$ to its tangent space. We consider the tangent space as a point in the space of $t$-dimensional planes in $\mathbb{R}^{e}$, the Grassmannian $\operatorname{Gr}(t, e)$, so we get a map $G: M \rightarrow \operatorname{Gr}(t, e)$. (The dashed arrow indicates that the map is a rational map: it is not defined at non-smooth points, which must lie on some subset of dimension less than $t$; formally, $G$ is defined on a Zariski open subset of $M$.) We may then consider the rank of $G$; in fact, this rank is closely related to $k_{\mathrm{sh}}$.

Proposition 4.6. The maps $G$ and $G \circ \ell$ both have rank $v d-k_{s h} d$.

Before proving this proposition, we make some preliminary definitions, paralleling the earlier definitions for the minimal stress kernel.

Definition 4.7. For $\rho \in C^{d}(\Gamma)$, define

$$
\begin{aligned}
& K(\rho)=\bigcap_{\Omega \in S(\rho)} \operatorname{ker}(\Omega) \\
& A(\rho)=\left\{\sigma \in C^{d}(\Gamma) \mid S(\rho) \subset S(\sigma)\right\} .
\end{aligned}
$$

Note that if $\Omega \in S(\rho)$, by definition $K(\rho) \subset K(\Omega)$ and $A(\rho) \subset A(\Omega)$. We can think of $K(\rho)$ as the 1-dimensional frameworks that satisfy every stress in $S(\rho)$. 
Lemma 4.8. The space $A(\rho)$ is isomorphic to $K(\rho)^{d}$.

Proof. By Lemma 2.11, we have

$$
A(\rho)=\bigcap_{\Omega \in S(\rho)} A(\Omega)=\bigcap_{\Omega \in S(\rho)} K(\Omega)^{d}=K(\rho)^{d} .
$$

Proof of Proposition 4.6. Fix a generic framework $\rho \in C^{d}(\Gamma)$. Observe that by Lemma 2.21, the equilibrium stresses for $\rho$ are the hyperplanes tangent to $\ell(\rho)$. By Lemma 2.21 again, these hyperplanes are also equilibrium stresses for any point $\sigma$ so that $\ell(\sigma)$ is smooth and such that $\ell(\sigma)$ is in the fiber of $G$ through $\ell(\rho)$. (But note that $\operatorname{rank}\left(d \ell_{\sigma}\right)$ need not be maximal.) Thus the fiber of $G \circ \ell$ through $\rho$ is contained in $A(\rho)$.

Now define the space $A^{\circ}(\rho)$ to be the set of frameworks $\sigma \in A(\rho)$ so that $\operatorname{rank}\left(d \ell_{\sigma}\right)$ is maximal and $\ell(\sigma)$ is a smooth point of $M$. Both conditions are algebraic, and $\rho$ satisfies both of them, so $A^{\circ}(\rho)$ is a non-empty Zariski open subset of $A(\rho)$. In particular, $\overline{A^{\circ}(\rho)}=A(\rho)$.

Meanwhile from Lemma 2.21 all points in $A^{\circ}(\rho)$ must be in the fiber of $G \circ \ell$.

Thus the fiber has dimension $\operatorname{dim} A^{\circ}(\rho)=\operatorname{dim} A(\rho)$. We therefore have

$$
\operatorname{dim} A(\rho)=\operatorname{dim} C^{d}(\Gamma)-\operatorname{rank}(G \circ \ell) .
$$

Since $G$ is defined on an open subset of the image of $\ell$, the image of $G$ is the same dimension as the image of $G \circ \ell$ and $\operatorname{rank}(G \circ \ell)=\operatorname{rank} G$. Putting these together with Lemma 4.8 we conclude

$$
\operatorname{rank}(G \circ \ell)=\operatorname{rank} G=\operatorname{dim} C^{d}(\Gamma)-\operatorname{dim} A(\rho)=v d-k_{\mathrm{sh}} d .
$$

In light of Proposition 4.6, since $k_{\mathrm{sh}} \geq d+1$, we have $\operatorname{rank} G \leq v d-(d+1) d$. Thus we can introduce the following terminology.

Definition 4.9. A graph $\Gamma$ has maximal Gauss rank in $\mathbb{E}^{d}$ if $\operatorname{rank} G=v d-(d+1) d$.

In particular, a graph $\Gamma$ has a minimal stress kernel iff it has maximal Gauss rank, and by Theorems 1.13, 1.14, and 4.4 both are equivalent to $\Gamma$ being generically globally rigid in $\mathbb{E}^{d}$.

4.2. Strengthening Connelly's proof. We now prove the missing direction of Theorem 4.4, mildly strengthening Connelly's proof of Theorem 1.13, closely following his argument.

Proof of Theorem 4.4. Let $\Gamma$ be a graph with $d+2$ or more vertices with $k_{\mathrm{sh}}(\Gamma, d)=d+1$. Let $\rho$ be a generic framework in $C^{d}(\Gamma)$, and let $\sigma$ be another framework with the same edge lengths. By [10, Proposition 3.3], there are neighborhoods $U_{\rho}$ and $U_{\sigma}$ of $\rho$ and $\sigma$ and a diffeomorphism $g: U_{\sigma} \rightarrow U_{\rho}$ with $\ell(g(x))=\ell(x)$ for $x \in U_{\sigma}$. In particular,

$$
\operatorname{span}\left(d \ell_{\sigma}\right)=\operatorname{span}\left(d \ell_{\rho}\right) \text {. }
$$

It follows that $S(\sigma)=S(\rho)$ (since both are equal to ann $\left(\operatorname{span}\left(d \ell_{\rho}\right)\right)$ ). Thus $\sigma$ is in $A(\rho)$, which is $K(\rho)^{d}$ by Lemma 4.8. By hypothesis, $K(\rho)$ consists of the coordinate projections of $\rho$ and the vector $\overrightarrow{1}$ of all 1's, so $\sigma$ is an affine image of $\rho$. (This is the analogue of $[10$, Theorem 4.1].)

As in the proof of [10, Theorem 1.3], for each vertex $v_{i}$ we can find a stress in $S(\rho)$ with some non-zero stress-value on an edge connected to the vertex. (Otherwise we could freely move $v_{i}$ without changing the space of equilibrium stresses, and get a stress kernel that is too large. Here we use the fact that there are at least $d+2$ vertices.) Therefore each vertex has degree at least $d+1$, as the vectors $v_{i}-v_{j}$ for $\{i, j\} \in \mathcal{E}$ are linearly dependent. Then 
by [10, Proposition 4.3], the edges of $\rho$ do not lie on a conic at infinity, so by [10, Proposition 4.2] any affine image of $\rho$ with the same edge lengths as $\rho$ is actually congruent to $\rho$. Therefore $\sigma$ is congruent to $\rho$, as desired.

4.3. Secant varieties and Zak's theorem. We add a few remarks about the algebraic geometry setting of these results. Both generic local rigidity and global rigidity relate to the geometry of the map $\ell$ and the measurement set. In both cases the appropriate rigidity property is characterized in terms of purely local behavior of the measurement set. For the generic local rigidity case, this is not surprising, but for the generic global rigidity case, it is a little unusual to be able to characterize a global property (the number of pre-images of a certain map) simply in terms of local behavior (the rank of the Gauss map).

Let us consider what happens as we vary the dimension $d$ while keeping the graph fixed. For the case $d=1$, the map $\ell$ is essentially an arbitrary quadratic map and the measurement set is of dimension $v-1$ if the graph is connected. Call this measurement set $M_{1}$, and denote by $\overline{M_{1}}$ its Zariski closure, which we call the measurement variety. (For the remainder of this section, we are interested in varying the ambient dimension while keeping the graph fixed, so we add a subscript.) In $d$ dimensions, the squared edge lengths are the sum of the squared edge lengths in each dimension separately. Thus the measurement variety $\overline{M_{d}}$ in $\mathbb{E}^{d}$ is the closure of the $d$-fold Minkowski sum of $\overline{M_{1}}$. Since $\overline{M_{1}}$ is a homogeneous variety, the Minkowski sum coincides with the secant variety, and so

$$
\overline{M_{d}}=\overline{\ell\left(C^{d}(\Gamma)\right)}=\overline{\sec ^{d}\left(\overline{M_{1}}\right)}
$$

where $\sec ^{d}\left(\overline{M_{1}}\right)$ is the $d$-fold secant variety of $\overline{M_{1}}$ (i.e., the join of $d$ copies of $\overline{M_{1}}$ ).

For general homogeneous varieties, the expected dimension of the join of two varieties is the sum of the two dimensions and so the expected dimension of the $d$-fold secant variety of a variety $V$ is $d$ times the dimension of $V$. If the dimension of the secant variety is less than the expected dimension the secant variety is said to be degenerate. In our case, because $\overline{M_{1}}$ is the closure of the image of a quadratic map there is automatically some degeneracy and its secant variety $\overline{M_{2}}$ has dimension at least one less than expected: $\operatorname{dim} \overline{M_{2}} \leq 2 \operatorname{dim} \overline{M_{1}}-1$. If our variety $\overline{M_{1}}$ were smooth or almost-smooth, Zak's Theorem on Superadditivity $[14,30]$ would imply that this degeneracy propagates, and given this first degeneracy, the $d$-fold secant $\overline{M_{d}}$ would have dimension at most $d \cdot \operatorname{dim} \overline{M_{1}}-\left(\begin{array}{l}d \\ 2\end{array}\right)$. Although the conditions of Zak's Theorem do not hold for a general graph $\Gamma$, the resulting bound is still exactly the dimension of $\overline{M_{d}}$ given by Theorem 1.6 for graphs that are generically locally rigid in $\mathbb{E}^{d}$. Thus generically locally rigid graphs are those where the secant variety is minimally degenerate in an appropriate sense.

Generic global rigidity instead involves another notion of degeneracy: the dual defect of a variety. For a general homogeneous variety $V$ in $\mathbb{R}^{n}$, the dual variety $V^{*}$ is $(n-1)$ dimensional. (In this case the generic contact locus $V_{\phi}$ is a single line through the origin.) The dual defect is defined to be $n-1-\operatorname{dim} V^{*}$ : the difference between the expected dimension of $V^{*}$ and the actual dimension. Again, in our setting $\overline{M_{d}}$ automatically has a dual defect of $\left(\begin{array}{c}d+1 \\ 2\end{array}\right)-1$ since it is the secant variety of the image of a quadratic map. Theorems 1.13 and 1.14 can be interpreted as saying that the graph is generically globally rigid if and only if the dual defect is this minimum.

Alternately, we can work with the Gauss defect. For a general homogeneous variety $V \subset$ $\mathbb{R}^{n}$, the rank of the Gauss map $G: V \rightarrow \operatorname{Gr}(t, n)$ is $\operatorname{dim} V-1$. (In fact, this happens whenever the projectivization $\mathbb{P} V$ is smooth.) Define the Gauss defect of $V$ to be $\operatorname{dim} V-$ 
$1-\operatorname{rank} G$. In general, the Gauss defect is less than or equal to the dual defect [15, Section 2.3.4]. Again, because $\overline{M_{d}}$ is the secant variety of the image of a quadratic map, the Gauss defect is at least $\left(\begin{array}{c}d+1 \\ 2\end{array}\right)-1$. By Theorem 4.4 the graph is generically globally rigid if and only if the Gauss defect is this minimum. (In particular, in this case the Gauss defect equals the dual defect.)

There is much literature on varieties with degenerate secant varieties or Gauss maps, although most of the work focuses on cases which are maximally, rather than minimally, degenerate $[16,22,31]$. From this point of view, Connelly's example [9] of $K_{5,5}$ (which is not generically globally rigid in 3 dimensions) is interesting. In this case the variety $\overline{M_{1}}$ is the cone on the Segre embedding of $\mathbb{R P}^{4} \times \mathbb{R} \mathbb{P}^{4}$ in $\mathbb{R P}^{9}$, and Connelly's result is about the degeneracy of the Gauss map of the third secant variety of this Segre embedding.

\section{Complexity of the Algorithm}

Implicit in Theorems 1.13 and 1.14 are a deterministic and a randomized algorithm. In this section, after briefly describing the deterministic algorithm (which is not very efficient), we will prove that testing whether a graph is generically globally rigid in $\mathbb{E}^{d}$ is in RP: that is, there is a polynomial-time randomized algorithm that will answer "no" all the time if the graph is not generically globally rigid in $\mathbb{E}^{d}$, and will answer "yes" at least half the time if the graph is generically globally rigid in $\mathbb{E}^{d}$. Hendrickson sketched an argument that testing for generic local rigidity is in RNC (which is contained in RP) [17, Section 2.2.2], and later sketched an randomized algorithm for determining whether a graph has a minimal stress kernel in $\mathbb{E}^{d}[18$, Section 2.3.1]. Here we will provide some more details on these algorithms, why they work, and what the necessary bounds on the size of the inputs are.

5.1. Deterministic algorithm. First we sketch the deterministic algorithm. Let $t:=v d-$ $\left(\begin{array}{c}d+1 \\ 2\end{array}\right)$ be the rank of the rigidity matrix for infinitesimally rigid frameworks, and let $s:=$ $v-d-1$ be the maximal rank of an equilibrium stress matrix for generic globally rigid frameworks.

Algorithm 5.1 (Deterministic check, global). To check if a graph $\Gamma$ with at least $d+2$ vertices is generically globally rigid in $\mathbb{E}^{d}$, take a $d$-dimensional framework with distinct coordinates, treated as independent formal symbols, and compute its rigidity matrix, again symbolic. By Gaussian elimination over the field of fractions of the coordinates, compute the rank of this matrix and kernel of its transpose. (The resulting kernel will be a ratio of polynomials in the symbolic inputs, and the answers will be valid for a numerical framework as long as none of the expressions in the denominators are zero.) If the rank of the rigidity matrix is not $t$, output the answer "no", as the graph is not even generically locally rigid. Otherwise take a generic equilibrium stress vector, i.e., a symbolic linear combination of a basis of the kernel of the transposed rigidity matrix, convert it to an equilibrium stress matrix, and compute its rank (again symbolically). If this rank is $s$, output the answer "yes", otherwise output the answer "no".

While this algorithm is guaranteed to give the correct answer, it is likely to be very slow, as the number of terms in the polynomials we need to manipulate can grow rapidly.

5.2. Efficient randomized algorithms. For a more efficient version, we turn to a similar randomized algorithm. 
Algorithm 5.2 (Randomized check, local). To check if a graph $\Gamma$ with at least $d+1$ vertices is generically locally rigid in $\mathbb{E}^{d}$, pick a framework $\rho$ in $C^{d}(\Gamma)$ with integer coordinates randomly chosen from $[1, N]$ for some suitably large $N$ (to be made precise below). Compute the rank of the rigidity matrix representing $d \ell_{\rho}$. If this rank is less than $t$, output "no", otherwise output "yes".

Algorithm 5.3 (Randomized check, global). To check if a graph $\Gamma$ with at least $d+1$ vertices is generically globally rigid in $\mathbb{E}^{d}$, proceed as follows. First, if the number of edges, $e$, is less than $t$ output "no" (as the graph cannot even be generically locally rigid with so few edges), otherwise continue.

Next pick a framework $\rho$ in $C^{d}(\Gamma)$ with integer coordinates randomly chosen from $[1, N]$ for some suitable $N$.

The next step is to pick one equilibrium stress vector $\omega$ from $S(\rho)$ in a suitably random way. Recall that an equilibrium stress vector is a vector in the annihilator of $\operatorname{span}\left(d \ell_{\rho}\right)$, i.e., in the kernel of $\left(d \ell_{\rho}\right)^{T}$. We find $\omega$ by setting up and solving an appropriate linear system $E \omega=b$ that extends the condition that $\omega \in \operatorname{ker}\left(\left(d \ell_{\rho}\right)^{T}\right)$. To create the linear system, create a matrix $H$ of $e-t$ random row vectors in $\mathbb{R}^{e}$, with coordinates integers chosen from $[1, N]$. Append these random rows to the transpose of the rigidity matrix to obtain an $e+\left(\begin{array}{c}d+1 \\ 2\end{array}\right)$ by $e$ matrix $E(\rho, H)$. Compute the rank of $E(\rho, H)$. If this rank is less than $e$, output "no", for either the rank of the rigidity matrix is less than $t$ (for instance if $\Gamma$ is not generically locally rigid), or $H$ contains some linear dependence with the rest of $E$, otherwise continue. (Note that, in constructing an RP algorithm, it is safe to output "no" in unfavorable cases, as long it is not done too often.)

Define $b$ to be a vector in $\mathbb{R}^{e+\left(\begin{array}{c}d+1 \\ 2\end{array}\right)}$ which is all zeros except for a single entry of 1 corresponding to one of the rows in $H$. (If $e=t$, there will be no such entry, $b$ will be all zeros and the only $\omega$ will be the zero vector.) Now solve the linear system $E \omega=b$, which must have exactly one solution, denoted $\omega(\rho, H)$.

Finally, convert $\omega(\rho, H)$ into an equilibrium stress matrix $\Omega(\rho, H)$, and compute its rank. If the rank is $s$, output the answer "yes", otherwise output the answer "no".

Remark 5.4. In the randomized algorithm as described above, the linear algebra computations are all done with explicit integer matrices. This can be done exactly in polynomial time using $p$-adic techniques [13]. (That is, first solve the equations modulo a prime $p$, then lift to a solution modulo $p^{2}$, and so forth.) However, since the algorithm is already randomized, the added complexity of this exact algorithm is unnecessary, and the computations can be done more simply by reducing modulo a suitably large prime $p$. See Proposition 5.11 below for a concrete estimate of how large the primes need to be.

To analyze the probability of the algorithm giving a false negative output, which depends on how large the parameter $N$ is, our main tool is the Schwartz-Zippel Lemma [12,27,32].

Lemma 5.5 (Schwartz, Zippel, DeMillo, Lipton). Let $\mathbf{k}$ be a field and $P \in \mathbf{k}\left[x_{1}, \ldots, x_{n}\right]$ be a non-zero polynomial of degree $d$ in the $x_{i}$. Select $r_{1}, \ldots, r_{n}$ uniformly at random from $a$ finite subset $X$ of $\mathbf{k}$. Then the probability than $P\left(r_{1}, \ldots, r_{n}\right)$ is 0 is less than $d /|X|$.

We also use the following basic principle:

Lemma 5.6. Let $M(\pi)$ be a matrix whose entries are polynomial functions with rational coefficients in the variables $\pi \in \mathbb{R}^{n}$. Let $r$ be a rank achieved by some $M\left(\pi_{0}\right)$. Then 
$\operatorname{rank}(M(\pi)) \geq r$ for all points $\pi$ that are generic in $\mathbb{R}^{n}$. More precisely, if the entries of $M(\pi)$ are polynomials of degree $g$, then there is a polynomial $P$ on $\mathbb{R}^{n}$ of degree $g \cdot r$ with rational coefficients so that $P(\pi)=0$ for any $\pi$ where the rank of $M(\pi)$ is less than $r$.

Proof. The rank of the $M(\pi)$ is less than $r$ iff the determinants of all of the $r \times r$ submatrices vanish. Let $\pi_{0} \in \mathbb{R}^{n}$ be a choice of parameters so $M\left(\pi_{0}\right)$ has rank $r$. Then there is an $r \times r$ submatrix $T\left(\pi_{0}\right)$ of $M\left(\pi_{0}\right)$ with non-zero determinant. Thus $\operatorname{det}(T(\pi))$ is a non-zero polynomial of $\pi$ of degree $g \cdot r$. For any $\pi$ with $\operatorname{rank}(M(\pi))<r$, this determinant must vanish. Thus, any such $\pi$ cannot be generic. We can therefore take $P(\pi)=\operatorname{det}(T(\pi))$.

We now apply these concepts to the simpler case of local rigidity for illustration.

Proposition 5.7. Algorithm 5.2 never returns a false "yes" answer, and returns a false "no" answer with probability bounded above by $t / N$. In particular, checking for generic local rigidity is in $R P$.

Proof. If the graph is not generically locally rigid, then from Lemma 5.6 the algorithm must always observe a rank which is less than $t$. Thus it will never give a false "yes" answer.

Suppose instead that $\Gamma$ is generically locally rigid in $\mathbb{E}^{d}$. The entries of the rigidity matrix are linear in $\rho$, so from Lemma 5.6 any $\rho$ which returns a false "no" answer must be at a zero of a polynomial of degree $t$ in our random variables. But by the Schwartz-Zippel Lemma, $\rho$ is a zero with probability less than $t / N$, as desired. If we choose $N>2 t$, we will give a false "no" answer less than $1 / 2$ the time.

The algorithms for finding ranks, etc., take time polynomial in $\log N$ (as well as the size of the matrix), so run in overall polynomial time. The one-sided nature of the errors means that the problem is in RP (rather than, say, BPP).

We now return to the global case. Recall that for a non-singular $n \times n$ matrix $M$,

$$
\operatorname{adj}(M)=\operatorname{det}(M) M^{-1},
$$

where $\operatorname{adj} M$ is the adjugate matrix of $M$, the conjugate of the cofactor matrix of $M$. In particular, adj $M$ is a polynomial in $M$ of degree $n-1$. This essentially means that, up to a global scale, the entries of the inverse of a matrix $M$ are polynomials in the entries of $M$. We will apply this to the matrix $E(\rho, H)$ from Algorithm 5.3 to see that the equilibrium stress matrix $\Omega(\rho, H)$ found by the algorithm can be though of as polynomials in the entries of $E(\rho, H)$. As the entries of $E(\rho, H)$ are polynomial functions in the coordinates of the framework $\rho$ and the random rows $H$, and matrix rank is invariant to scaling, this will put us in position to apply Lemma 5.6.

There are a few technical issues that must be dealt with in this analysis. Most importantly, the matrix $E(\rho, H)$ has more rows than columns. We will deal with this by looking at an appropriate square submatrix of $E(\rho, H)$, as follows.

Lemma 5.8. Let $\Gamma$ be a graph, and let $t^{\prime}$ be the maximal value of rank $d \ell_{\rho}$ over all frameworks $\rho$ of $\Gamma$ in $\mathbb{E}^{d}$. (For generically locally rigid graphs $t^{\prime}=t$.) Let $U$ be the set of $\rho$ where this maximal rank is achieved. Let $s^{\prime}$ be the maximal value of $\operatorname{rank}(\Omega)$ over all stress matrices of all $\rho$ in $U$. Then for all generic $\rho$, we have $\max _{\Omega \in S(\rho)} \operatorname{rank}(\Omega)=s^{\prime}$.

More precisely, there is a polynomial $P$ of degree $e+s^{\prime}(e-1)$ in the variables $(\rho, H)$, where $H$ is a set of additional rows as in Algorithm 5.3, so that if $(\rho, H)$ is not a zero of $P$ then the stress matrix $\Omega(\rho, H)$ is well-defined and has rank $s^{\prime}$. 
Here, as in Algorithm 5.3, for each $\rho$ we add a set $H$ of $e-t^{\prime}$ additional rows in $\mathbb{R}^{e}$ to the transpose of the rigidity matrix of $\rho$ to define a matrix $E(\rho, H)$, and find an equilibrium stress vector $\omega(\rho, H)$ by solving the linear system $E \omega=b$ where $b \in \mathbb{R}^{e}$ is a vector of all zeroes except for a single 1 in one of the positions of a row in $H$ (if any). This is then converted to a stress matrix $\Omega(\rho, H)$. The assertion that $\Omega(\rho, H)$ is well-defined means that this linear system has a unique solution. (In particular, this implies that $\rho \in U$.)

Proof of Lemma 5.8. Let $\rho_{0} \in U$ be a framework with an equilibrium stress $\omega_{0}$ so that the corresponding stress matrix $\Omega_{0}$ has rank $s^{\prime}$. Find a set $H_{0}$ of additional rows so that $E\left(\rho_{0}, H_{0}\right)$ has rank $e$ and $E\left(\rho_{0}, H_{0}\right) \omega_{0}=b$. Let $\hat{E}(\rho, H)$ be an $e \times e$ submatrix of $E(\rho, H)$ so that $\hat{E}\left(\rho_{0}, H_{0}\right)$ is invertible. ( $\hat{E}$ necessarily consists of $t^{\prime}$ rows from the transpose of the rigidity matrix and all the rows of $H$.) Define $\hat{b}$ similarly, let $\tilde{\omega}(\rho, H):=\operatorname{adj}(\hat{E}) \hat{b}$, and let $\tilde{\Omega}(\rho, H)$ be the associated stress matrix.

By Lemma 5.6, the rank of $\hat{E}(\rho, H)$ is equal to its maximum value $e$ at all points $(\rho, H)$ that are not zeros of a polynomial $P_{1}(\rho, H)=\operatorname{det} \hat{E}(\rho, H)$, which has degree $e$. Moreover, when $P_{1}(\rho, H) \neq 0$, the linear equation defining $\Omega$ has a unique solution and the adjugate matrix $\tilde{\Omega}(\rho, H)$ is a scalar multiple of $\Omega(\rho, H)$. In particular we have assumed $\left(\rho_{0}, H_{0}\right)$ is not a zero of $P_{1}$ and thus $\tilde{\Omega}\left(\rho_{0}, H_{0}\right)$ has rank $s^{\prime}$. By Lemma 5.6 again, the rank of $\tilde{\Omega}(\rho, H)$ is less than $s^{\prime}$ only at the zeros of a non-zero polynomial $P_{2}(\rho, H)$ of degree $s^{\prime}(e-1)$ (as the entries of $\tilde{\Omega}(\rho, H)$ have degree $e-1$ in $(\rho, H))$.

For any generic $\rho$, there must be some generic point $(\rho, H)$. At such a generic $(\rho, H)$ $\tilde{\Omega}(\rho, H)$ and $\Omega(\rho, H)$ must have rank not less than $s^{\prime}$. Meanwhile we have supposed that rank of such $\Omega(\rho, H)$ is never larger than $s^{\prime}$, and thus have proven our claim.

In particular, let $P=P_{1} \cdot P_{2}$. Then if $(\rho, H)$ is not a zero of $P$, the linear equation $E \omega=b$ has a unique solution (as $P_{1} \neq 0$ ) and the resulting stress matrix $\Omega(\rho, H)$ has rank $s^{\prime}$ (as $\left.P_{2} \neq 0\right)$.

Remark 5.9. In fact, since the matrix rank drops only at the zeros of an algebraic equation, we can prove the stronger statement that all generic $\Omega \in S(\rho)$ of all generic $\rho$ have $\operatorname{rank}(\Omega)=s^{\prime}$. Here we consider $S(\rho)$ as defined over the smallest extension of $\mathbb{Q}$ containing $\rho$.

The following theorem is a more precise version of Theorem 1.17.

Theorem 5.10. Algorithm 5.3 never returns a false "yes" answer, and returns a false "no" answer with probability bounded above by ve/N. In particular, checking for generic global rigidity in $\mathbb{E}^{d}$ is in $R P$.

Proof. First we suppose that $\Gamma$ is not generically globally rigid in $\mathbb{E}^{d}$. If the rigidity matrix does not have rank $t$, then the algorithm correctly outputs "no". If the rigidity matrix does have rank $t$, by Lemma 5.8, the maximal observed rank cannot be larger than the rank of any equilibrium stress matrix of any generic framework, which is less than $s$ by supposition. Thus the algorithm will correctly output "no".

Next we suppose that $\Gamma$ is generically globally rigid in $\mathbb{E}^{d}$ and bound the probability of a false negative.

Let $(\rho, H)$ be a set of variables that are not a zero of the polynomial $P$ given by Lemma 5.8. Such points cannot give a false negative: the rank of $E(\rho, H)$ is its maximum value, $e$, so we do not give a false negative in constructing $\omega$, and $\operatorname{rank}(\Omega)=s$, so again we do not output a false negative. 
So if $\Gamma$ is generically globally rigid in $\mathbb{E}^{d}$, we are guaranteed to obtain the correct answer if we pick $\rho$ and $H$ that miss the zeros of $P$, a polynomial of degree $e+s(e-1)$, which is less than ve. The Schwartz-Zippel Lemma, Lemma 5.5, then gives the bounds stated on the error probability. Moreover, if we choose $N>2 v e$, we will give a false "no" answer less than half the time.

The running time for such an $N$ is polynomial in the input size, so testing for generic global rigidity is in $\mathrm{RP}$.

Algorithm 5.3 and the proof of Theorem 5.10 are structured as they are because Lemma 5.8 is false without the condition that rank $d \ell_{\rho}$ take its maximal value. For instance, if a framework $\rho$ lies in a lower-dimensional subspace of $\mathbb{E}^{d}$, the rank of a generic $\Omega \in S(\rho)$ may be greater than $v-d-1$, so the universal upper bound motivating Definition 1.12 may not hold.

5.3. Estimating the primes. For the reader's convenience, we now estimate some bounds on the size of the primes necessary for the algorithms to work with high probability. These bounds can very likely be improved substantially.

Proposition 5.11. Suppose that the computations in Algorithm 5.2 or 5.3 are done modulo a prime $p$ then neither algorithm will return a false "yes" answer. If the prime is chosen chosen uniformly from a set of primes $R$ with each prime larger than $N$, and if $|R|>4 t$ and $N>4 t$, then Algorithm 5.2 produces a false "no" answer with probability bounded above by 1/2. Similarly, if $|R|>4$ ve and $N>4$ ve, then Algorithm 5.3 produces a false "no" answer with probability bounded above by $1 / 2$.

Remark 5.12. By the Prime Number Theorem, this proposition tells us we need to consider primes up to about $8 t \ln (4 t)$ (for local rigidity) or $8 v e \ln (4 v e)$ (for global rigidity) in order to get a sufficiently large set $R$.

Proof. Doing the computations modulo a prime can only make the computed ranks drop, so as before, we cannot return a false "yes" answer.

If the graph is generically locally (or globally) rigid, there is an integer polynomial $P_{\text {loc }}$ (or $\left.P_{\text {glob}}\right)$ so that if our random framework $\rho$ (or pair $(\rho, H)$ ) is not a zero of $P_{\text {loc }}$ (or $P_{\text {glob }}$ ) we do not return a false "no" answer. The Schwartz-Zippel Lemma, Lemma 5.5, works without change modulo $p$. The only differences are that

- the prime $p$ must be large enough so that there are enough distinct values modulo $p$ for the lemma to be useful, and

- the polynomial $P$ not be zero modulo $p$.

To take care of the first point, we require that the prime be larger than the chosen value $N$ in the Schwartz-Zippel Lemma.

To take care of the second point, we will give an upper bound $B$ for the sum of the absolute values of the coefficients of $P$. If we pick a prime $p$ larger than $B$ (or in fact larger than the absolute value of any single coefficient of $P$ ) then $P$ will be guaranteed to be non-zero modulo $p$. However, we can in fact randomly pick a smaller prime from a suitable collection, and still guarantee that with high probability $P$ will be non-zero modulo $p$. In particular, suppose that we have a collection of primes $R$ so that any product of at least $|R| / 4$ primes in the collection is larger than $B$. Then, any subset of size at least $|R| / 4$ must have some prime such that $P$ is non-zero modulo $p$. (Otherwise, $P$ would have to be zero modulo this product, but this this product is larger than $B$.) Thus $P$ is zero modulo $p$ for at most $1 / 4$ of 
the primes in $R$. Then if we pick $p$ at random from $R$, the chance that $P$ is zero modulo $p$ is at most $1 / 4$.

We now estimate the sum of the coefficients of $P$ in the two cases. For local rigidity, $P_{\text {loc }}$ is a determinant of a $t \times t$ submatrix of the rigidity matrix. As such, it is a sum of $t$ ! terms, with each term a product of $t$ factors, and with each factor having coefficient-sum equal to 2 . Using the estimate $n !<n^{n}$, we therefore find

$$
\ln B_{\mathrm{loc}} \leq \ln \left(2^{t} \cdot t !\right)<t(\ln t+\ln 2) .
$$

For global rigidity, we separately estimate bounds $B_{1}, B_{2}$ for the two factors $P_{1}, P_{2}$ making up $P_{\text {glob }}$ from the proof of Lemma 5.8. We find

$$
\ln B_{1} \leq \ln \left(2^{e_{\Gamma}} \cdot e_{\Gamma} !\right)<e_{\Gamma}\left(\ln e_{\Gamma}+\ln 2\right) .
$$

(Here we write $e_{\Gamma}$ for the number of edges in $\Gamma$ to avoid confusion with the base of natural logarithms.) Note that $P_{2}$ is an $s \times s$ determinant of $\tilde{\Omega}$, which itself is made of $\left(e_{\Gamma}-1\right) \times\left(e_{\Gamma}-1\right)$ determinants of $E$. We therefore have

$$
\ln B_{2} \leq \ln \left(s !\left(2^{e_{\Gamma}} e_{\Gamma} !\right)^{s}\right)<s \ln s+s e_{\Gamma} \ln 2+s e_{\Gamma} \ln e_{\Gamma} .
$$

Now suppose, in the local rigidity case, we pick a set of primes $R$ with $|R|>4 t$ and each prime in $R$ larger than $4 t$. Then, for any subset $R^{\prime}$ of $R$ of size at least $t, \prod_{p \in R^{\prime}} p>B_{\text {loc }}$. Indeed,

$$
\sum_{p \in R^{\prime}} \ln p-\ln B_{\mathrm{loc}} \geq t \ln (4 t)-t(\ln t+\ln 2)=t \ln 2>0 .
$$

Therefore if we pick a prime $p$ randomly from $R, P_{\text {loc }}$ is non-zero modulo $p$ with probability at least $3 / 4$, and the Schwartz-Zippel Lemma tells us that if $P_{\text {loc }}$ is non-zero modulo $p$ we (correctly) answer "yes" with probability at least $3 / 4$. We conclude that our total probability of returning the correct answer is at least $\frac{3}{4} \cdot \frac{3}{4}>\frac{1}{2}$, as desired.

Similarly, for global rigidity, we find

$$
\begin{aligned}
\ln B_{\text {glob }} \leq \ln B_{1}+\ln B_{2} & \leq(s+1) e_{\Gamma} \ln e_{\Gamma}+(s+1) e_{\Gamma} \ln 2+s \ln s \\
& <v e_{\Gamma} \ln e_{\Gamma}+v e_{\Gamma} \ln 2+v \ln v \\
& <v e_{\Gamma} \ln \left(4 v e_{\Gamma}\right) .
\end{aligned}
$$

Again, suppose we pick a set of primes $R$ with $|R|>4 v e_{\Gamma}$ and each prime bigger than $4 v e_{\Gamma}$. Then for any $R^{\prime} \subset R$ with $\left|R^{\prime}\right|>|R| / 4$, we have $\prod_{p \in R^{\prime}} p<B_{\text {glob }}$, as desired.

\section{Smooth HigheR-Dimensional FLEXES}

Let us now turn to the issue of higher-dimensional flexing. Given incongruent frameworks $\rho$ and $\sigma$ in $\mathbb{E}^{d}$ with the same edge lengths, how large must $a$ be so that $\rho$ and $\sigma$ are connected by a smooth path of frameworks in $\mathbb{E}^{d+a}$ with constant edge lengths? (Such a path is called a flex in $\mathbb{E}^{d+a}$.) Bezdek and Connelly [4] have shown that an arbitrary pair of frameworks in $\mathbb{E}^{d}$ with the same edge lengths can be connected by a smooth path in $\mathbb{E}^{2 d}$ (so $a=d$ always suffices), while Belk and Connelly [3] exhibited a $d$-dimensional framework of the " $d$-simplex with flaps" which is not globally rigid in $\mathbb{E}^{d}$ but still locally rigid all the way up to $\mathbb{E}^{2 d-1}$ (so $a=d$ can be necessary). Thus for arbitrary frameworks one may have to go up to twice the dimension to get any flexibility at all. Theorem 1.18, which we will now prove, states that the situation is rather different for generic frameworks: for a generic framework $\rho$ which is not globally rigid, there is some other incongruent framework $\sigma$ so that $\rho$ can be flexed to $\sigma$ 
in $\mathbb{E}^{d+1}$ (so $a=1$ suffices). The question of how large $a$ must be to reach every alternative framework with the same edge lengths as a given generic one remains open.

The rest of this section is devoted to the proof of Theorem 1.18. Note that for the conditions of the theorem to be satisfied $\Gamma$ must have at least $d+2$ vertices and must not have a minimal stress kernel.

Definition 6.1. Given a framework $\rho \in C^{d}(\Gamma)$, and a stress $\Omega \in S(\rho)$, the space of lifted stress satisfiers $\widetilde{A}(\Omega)$ is the space of all $(d+1)$-dimensional frameworks of $\Gamma$ that satisfy $\Omega$. Also let $\tilde{\rho}$ be the framework in $\widetilde{A}(\Omega)$ defined by embedding $\rho$ in the first $d$ dimensions of $\mathbb{E}^{d+1}$. In our proof, our $(d+1)$-dimensional flex will in fact stay in the space $\widetilde{A}(\Omega)$. Because $\widetilde{A}(\Omega)$ is isomorphic to $K(\Omega)^{d+1}$, it is a $(k d+k)$-dimensional linear space, where $k:=\operatorname{dim}(K(\Omega))$. Finally, let $\widetilde{\ell}$ be the length squared map on $\widetilde{A}(\Omega)$ and define $\widetilde{B}(\Omega):=\widetilde{\ell}(\widetilde{A}(\Omega))$.

We summarize here the main spaces and maps that will be used in this proof.

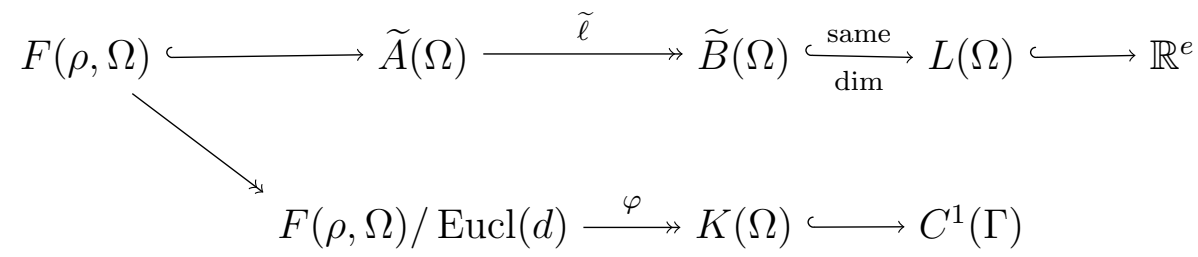

Let $F(\rho, \Omega)$ be $\tilde{\ell}^{-1}(\ell(\rho))$, the fiber of $\tilde{\ell}$ over the point $\ell(\rho)$, and let $F_{0}(\rho, \Omega)$ be the connected component containing $\tilde{\rho}$. Thus $F(\rho, \Omega)$ consists of points in $\widetilde{A}(\Omega)$ with the same edge lengths as $\rho$. We will first show that for any generic $\rho$, and suitably generic $\Omega \in S(\rho), F(\rho, \Omega)$ is a smooth manifold. To do this, we will need to know that $\ell(\rho)$ is a regular value of $\tilde{\ell}$ (Lemma 6.3). To compute the dimension of $F(\rho, \Omega)$, we will show that $\widetilde{B}(\Omega)$ is the same dimension as the space $L(\Omega)$, which was defined in Definition 2.14.

By definition, $\tilde{\rho}$ has all zeros in its last $((d+1)$ 'st $)$ coordinate. Thus our plan is to show that there is another incongruent framework in $F_{0}(\rho, \Omega)$ that also has all zeros in its last coordinate and is thus in $C^{d}(\Gamma)$. To deal with congruences, we will mod out by the Euclidean transformations in the first $d$ coordinates. When $\Gamma$ does not have a minimal stress kernel, the singularities of $F_{0}(\rho, \Omega) / \operatorname{Eucl}(d)$ will be of codimension 2 or greater (Lemma 6.6; compare Proposition 2.13). We then consider a proper map $\varphi$ from $F_{0}(\rho, \Omega) / \operatorname{Eucl}(d)$ that looks at the last coordinate of a framework, so $[\tilde{\rho}]$ maps to 0 . We will prove that this map has a well defined and even degree. This will prove the existence of a second framework $\tilde{\sigma}$ in $F_{0}(\rho, \Omega)$ which maps to zero under $\varphi$ and thus comes from another framework $\sigma$ in $C^{d}(\Gamma)$ with the same edge lengths.

Since $\tilde{\rho}$ and $\tilde{\sigma}$ are both in $F_{0}(\rho, \Omega)$, which is connected by assumption, there must be a smooth path within $F(\rho, \Omega)$ which connects the two frameworks, which is the desired smooth flex in $\mathbb{E}^{d+1}$.

We now proceed to fill in the details. In order to understand the fibers of $\widetilde{\ell}$, we would like to apply Sard's Theorem, but we first need to know that $\ell(\rho)$ is a generic point in $L(\Omega)$ in an appropriate sense. Here we use the standard notation $\mathbb{Q}(x)$, where $x \in \mathbb{R}^{n}$, to mean the smallest field containing $\mathbb{Q}$ and all the coordinates of $x$.

Lemma 6.2. Let $\rho \in C^{d}(\Gamma)$ and $\omega \in S(\rho)$ such that $(\ell(\rho), \omega)$ is generic in $C_{\bar{M}}$. Then $\ell(\rho)$ is generic inside $L(\Omega)$, where we consider $L(\Omega)$ to be defined over $\mathbb{Q}(\Omega)$. 
Proof. Suppose that $\ell(\rho)$ is not generic in $B(\Omega)$ over $\mathbb{Q}(\Omega)$. Then, by the definition of genericity, there is some polynomial function $P$ with coefficients in $\mathbb{Q}$ so that $P(\ell(\rho), \Omega)=0$ while there is some $\rho^{\prime} \in A(\Omega)$ so that $P\left(\ell\left(\rho^{\prime}\right), \Omega\right) \neq 0$. We can suppose that $d \ell_{\rho^{\prime}}$ has maximal rank and $\ell\left(\rho^{\prime}\right)$ is smooth in $\bar{M}$ since having non maximal rank, having an image under $\ell$ that is non smooth in $\bar{M}$, and the vanishing of $P$ are all defined by algebraic equations that do not vanish identically on $A(\Omega)$. Since $\Omega$ is an equilibrium stress for $\rho^{\prime}, d \ell_{\rho^{\prime}}$ has maximal rank, and $\ell\left(\rho^{\prime}\right)$ is smooth, from Lemma $2.21\left(\ell\left(\rho^{\prime}\right), \omega\right)$ must be in $C_{\bar{M}}$. Thus $P$ is a polynomial function that does not vanish identically over $C_{\bar{M}}$, contradicting the genericity of $(\ell(\rho), \omega)$ in $C_{\bar{M}}$.

Since $\ell(\rho)$ is generic in $B(\Omega)$, it is also generic in its Zariski closure $L(\Omega)$.

Next we can look at the map $\tilde{\ell}$ and its fibers. Let $F(\rho, \Omega):=\tilde{\ell}^{-1}(\ell(\rho))$ be the fiber of $\tilde{\ell}$ at the point $\ell(\rho)$. Also let $F_{0}(\rho, \Omega)$ be the component of $F(\rho, \Omega)$ that includes $\rho$.

Lemma 6.3. Suppose $(\ell(\rho), \omega)$ is generic in $C_{\bar{M}}$. Then $F(\rho, \Omega)$ is a smooth manifold.

Proof. From Lemma 6.2, $\ell(\rho)$ is a generic point in $L(\Omega)$. Thus from the algebraic version of Sard's Theorem over the field $\mathbb{Q}(\Omega), \ell(\rho)$ is a regular value of $\widetilde{\ell}$. Therefore, from the implicit function theorem, $F(\rho, \Omega)$ is a smooth manifold.

Lemma 6.4. Suppose that $\Gamma$, a graph with $d+2$ or more vertices, is generically locally rigid in $\mathbb{E}^{d}$ and $(\ell(\rho), \omega)$ is generic in $C_{\bar{M}}$. Then the semi-algebraic set $\widetilde{B}(\Omega)$ has the same dimension as $B(\Omega)$ and $L(\Omega)$, namely $k d-\left(\begin{array}{c}d+1 \\ 2\end{array}\right)$.

Proof. In one direction, $\widetilde{B}(\Omega) \supset B(\Omega)$ and so clearly is at least of dimension $k d-\left(\begin{array}{c}d+1 \\ 2\end{array}\right)$. Recall from Proposition 2.23 that $B(\Omega)$ is a flat space and so is contained in $L(\Omega)$, a linear space of the same dimension. In the other direction, we need to show that $\widetilde{B}(\Omega)$ is contained in $L(\Omega)$. As described in Lemma 2.26, $B(\Omega)$ coincides with $\operatorname{chord}^{d}(\ell(K(\Omega)))$ and similarly $\widetilde{B}(\Omega)$ is $\operatorname{chord}^{d+1}(\ell(K(\Omega)))$. But then $\widetilde{B}(\Omega) \subset \operatorname{chord}^{2 d}(\ell(K(\Omega)))=\operatorname{chord}^{2}(B(\Omega)) \subset L(\Omega)$, as desired.

Corollary 6.5. Suppose that $\Gamma$, a graph with $d+2$ or more vertices, is generically locally rigid in $\mathbb{E}^{d}$ and $(\ell(\rho), \omega)$ is generic in $C_{\bar{M}}$. Then $F(\rho, \Omega)$ is a smooth manifold of dimension $k+\left(\begin{array}{c}d+1 \\ 2\end{array}\right)$.

Proof. $F(\rho, \Omega)$ is a smooth manifold by Lemma 6.3. To find its dimension, subtract the dimension of the image from the dimension of the domain:

$$
\operatorname{dim}(F(\rho, \Omega))=\operatorname{dim} \widetilde{A}(\Omega)-\operatorname{dim} \widetilde{B}(\Omega)=k(d+1)-\left(k d-\left(\begin{array}{c}
d+1 \\
2
\end{array}\right)\right)=k+\left(\begin{array}{c}
d+1 \\
2
\end{array}\right) .
$$

Next we mod out by the the group consisting of Euclidean transformations on the first $d$ coordinates. (We do not mod out by Euclidean transforms involving the last coordinate, as we want to be able to detect when the framework lies completely in $\mathbb{E}^{d}$.) This gives us the quotient $F(\rho, \Omega) / \operatorname{Eucl}(d)$.

Lemma 6.6. Suppose that $\Gamma$, a graph with $d+2$ or more vertices, is generically locally rigid in $\mathbb{E}^{d},(\ell(\rho), \Omega)$ is generic in $C_{\bar{M}}$, and $k>d+1$. Then $F(\rho, \Omega) / \operatorname{Eucl}(d)$ is a smooth stratified space of dimension $k$ with singularities of codimension at least 2 .

Proof. From Corollary 6.5, $F(\rho, \Omega)$ is a smooth manifold. So by the stratified structure on quotient spaces as in Lemma 2.12, $F(\rho, \Omega) / \operatorname{Eucl}(d)$ is a smooth stratified space with 
singularities at quotients with non-trivial stabilizer, i.e., frameworks whose projection down to the first $d$ coordinates span a proper affine subspace of $\mathbb{E}^{d}$.

First we argue that such singularities occur only at frameworks that span exactly a $d$ dimensional subspace of $\mathbb{E}^{d+1}$ and that project down to subspace of dimension exactly $d-1$ in the first $d$ coordinates. In particular, such singularities cannot have an affine span of less than $d$ in $\mathbb{E}^{d+1}$. Any framework with a smaller affine span would give a framework in $\mathbb{E}^{d^{\prime}}$ for some $d^{\prime}<d$. Considered as a framework in $\mathbb{E}^{d}$, this would mean that $\ell(\rho)$ is not a regular value of $\ell$, contradicting Lemma 2.39. Thus the only possibility for a larger-thanexpected stabilizer is a framework with $d$-dimensional span whose projection is smaller than $d$-dimensional, as claimed.

The dimension of these singular frameworks is $d+\left(\begin{array}{c}d+1 \\ 2\end{array}\right)$ : it is $d$ (the dimension of the space of hyperplanes in $\mathbb{E}^{d}$, the possible choices of $(d-1)$-dimensional hyperplanes) plus $\left(\begin{array}{c}d+1 \\ 2\end{array}\right)$ (the dimension of $\operatorname{Eucl}(d)$, the possible choices of the framework within a single hyperplane; here we use local rigidity in $\mathbb{E}^{d}$ and the fact that $\ell(\rho)$ is a regular value of $\ell$ ). The singular frameworks are therefore codimension $k-d$ inside $F(\rho, \Omega)$.

Since the stabilizer of these singular frameworks is always $O(1) \simeq \mathbb{Z} / 2$, which is discrete, the codimension of the singular set does not change inside the quotient. In particular, since $\Gamma$ does not have a minimal stress kernel, the codimension is always at least 2 .

Since generically the stabilizer is trivial, the dimension of the quotient $F(\rho, \Omega) / \operatorname{Eucl}(d)$ is $\operatorname{dim}(F(\rho, \Omega))-\operatorname{dim}(\operatorname{Eucl}(d))=k$.

Now we will look at the last coordinate of frameworks in $F_{0}(\rho, \Omega) / \operatorname{Eucl}(d)$. Since all such frameworks are in $\widetilde{A}(\Omega)$, this coordinate must be in $K(\Omega)$. (Here we are thinking of $K(\Omega$ ) as a space of frameworks in $\mathbb{E}^{1}$.) Points that map to 0 under this map represent classes of frameworks that lie entirely in the space spanned by the first $d$ coordinates in $\mathbb{E}^{d+1}$. Like all frameworks in $F(\rho, \Omega)$, they have the same edge lengths as $\rho$.

Definition 6.7. Let the map $\varphi$ from $F_{0}(\rho, \Omega) / \operatorname{Eucl}(d)$ to $K(\Omega)$ be given by simply looking at the last coordinate.

Lemma 6.8. The map $\varphi: F_{0}(\rho, \Omega) / \operatorname{Eucl}(d) \rightarrow K(\Omega)$ is proper.

Proof. Think of $F_{0}(\rho, \Omega) / \operatorname{Eucl}(d)$ as a subset of the product $C^{d}(\Gamma) / \operatorname{Eucl}(d) \times C^{1}(\Gamma)$. It suffices to show that $\varphi^{-1}(P)$ is bounded for any compact subset $P$ of $K(\Omega)$. (It is automatic that $\varphi^{-1}(P)$ is closed.) Inside $C^{d}(\Gamma) / \operatorname{Eucl}(d)$, all of $F_{0}(\rho, \Omega) / \operatorname{Eucl}(d)$ is bounded (as the edge lengths are bounded by the edge lengths of $\rho$ ). On the other hand, since $P$ is a bounded subset of $K(\Omega)$, the projection of $\varphi^{-1}(P)$ to $C^{1}(\Gamma)$, which is just $P$ itself, is by supposition bounded.

(Compare Lemma 2.34.)

And now we are in position to complete the proof of our Theorem.

Proof of Theorem 1.18. If $\Gamma$ is not generically locally rigid in $\mathbb{E}^{d}$, then clearly generic frameworks can be flexed in $\mathbb{E}^{d+1}$ (as they can be flexed in $\mathbb{E}^{d}$ ). So from now on we assume $\Gamma$ is generically locally rigid in $\mathbb{E}^{d}$.

By Lemma 2.24, for any generic $\rho$, there is an $\omega \in S(\rho)$ such that $(\ell(\rho), \omega)$ is generic in $C_{\bar{M}}$. Choose this $\omega$ to define $\varphi$. Since $\Gamma$ does not have a minimal stress kernel, $k \geq k_{\min }>d+1$. (In fact for such a generic $\Omega, k$ must equal $k_{\min }$ ).

By Lemma 6.6, when $k>d+1$ the space $F_{0}(\rho, \Omega) / \operatorname{Eucl}(d)$ has singularities of high codimension. By Lemma 6.8, $\varphi$ is proper, and by Lemma 6.6 again, the domain of $\varphi$ has 
the same dimension as its range (which is $k$ ). Thus $\varphi$ has a well-defined mod-two degree by Corollary 2.36. The frameworks in $F_{0}(\rho, \Omega)$ have fixed edge lengths, which implies that the image frameworks in $K(\Omega)$, considered as a subset of $C^{1}(\Gamma)$, have all points within a bounded distance of each other. In particular $\varphi$ is not onto, and so its mod-two degree must be zero. The preimages of 0 are congruence classes of frameworks of $\Gamma$ that lie in $\mathbb{E}^{d}$ and have the same edge lengths as $\rho$.

Next, by the analysis in Lemma 6.6, the point 0 cannot be the image of a singularity of $F_{0}(\rho, \Omega) / \operatorname{Eucl}(d)$. Furthermore, 0 is a regular value of $\varphi$ : An element of the kernel of $d \varphi$ at $[\sigma]$ for some $\sigma$ in the inverse image of 0 is an infinitesimal $d$-dimensional flex of the framework $\sigma$ in $C^{d}(\Gamma)$. But Lemma 2.39 tells us that every framework in $C^{d}(\Gamma)$ with the same edge lengths as $\rho$ is infinitesimally rigid. Thus there are an even number of points in $\varphi^{-1}(0)$. Let $[\tilde{\sigma}]$ be another such point. Since $\tilde{\rho}$ and $\tilde{\sigma}$ are points in a connected smooth manifold, there is a smooth path connecting them. This smooth path is the desired path of frameworks of $\Gamma$ in $\mathbb{E}^{d+1}$ with constant edge lengths.

\section{REFERENCES}

[1] L. Asimow and B. Roth, The rigidity of graphs, Trans. Amer. Math. Soc. 245 (1978), 279-289.

[2] _ The rigidity of graphs, II, J. Math. Anal. Appl. 68 (1979), 171-190.

[3] M. Belk and R. Connelly, Rigidity of the simplex with flaps, preprint, 2007.

[4] K. Bezdek and R. Connelly, The Kneser-Poulsen conjecture for spherical polytopes, Discrete Comput. Geom. 32 (2004), no. 1, 101-106.

[5] J. Bochnak, M. Coste, and M. F. Roy, Real algebraic geometry, Springer, 1998.

[6] E. D. Bolker and B. Roth, When is a bipartite graph a rigid framework?, Pacific J. Math. 90 (1980), no. $1,27-44$.

[7] S. Capkun, M. Hamdi, and J. P. Hubaux, GPS-free positioning in mobile ad hoc networks, Cluster Computing 5 (2002), no. 2, 157-167.

[8] M. Cheung and W. Whiteley, Transfer of global rigidity results among dimensions: Graph powers and coning, Preprint, York University, 2005.

[9] R. Connelly, On generic global rigidity, DIMACS Ser. Discrete Math. Theoret. Comput. Sci. 4 (1991), $147-155$

[10] Generic global rigidity, Discrete Comput. Geom 33 (2005), no. 4, 549-563.

[11] G. M. Crippen and T. F. Havel, Distance geometry and molecular conformation, Taunton, 1988.

[12] R. DeMillo and R. Lipton, A probabilistic remark on algebraic program testing, Information Processing Letters 7 (1978), no. 4, 192-194.

[13] J. D. Dixon, Exact solution of linear equations using p-adic expansions, Numer. Math. 40 (1982), 137141.

[14] B. Fantechi, On the superadditivity of secant defects, Bull. Soc. Math. France 118 (1990), no. 1, 85-100.

[15] G. Fischer and J. Piontkowski, Ruled varieties: An introduction to algebraic differential geometry, Advanced Lectures in Mathematics, Friedrick Vieweg \& Sohn, Braunschweig, 2001.

[16] W. Fulton and R. Lazarsfeld, Connectivity and its applications in algebraic geometry, Algebraic Geometry (Chicago, Ill., 1980), Lecture Notes in Math., vol. 862, Springer, 1981, pp. 26-92.

[17] B. Hendrickson, Conditions for unique graph realizations, SIAM J. Comput. 21 (1992), no. 1, 65-84.

[18] _ The molecule problem: Exploiting structure in global optimization, SIAM J. Optim. 5 (1995), no. 4, 835-857.

[19] B. Jackson and T. Jordán, Connected rigidity matroids and unique realizations of graphs, J. Combin. Theory Ser. B 94 (2005), no. 1, 1-29.

[20] B. Jackson, T. Jordán, and Z. Szabadka, Globally linked pairs of vertices in equivalent realizations of graphs, Discrete Comput. Geom. 35 (2006), no. 3, 493-512.

[21] G. Laman, On graphs and rigidity of plane skeletal structures, J. Engrg. Math. 4 (1970), no. 4, 331-340.

[22] R. Lazarsfeld and A. V. de Ven, Topics in the geometry of projective space: Recent work of F. L. Zak, DMV Seminar, vol. 4, Birkhäuser Verlag, Basel, 1984, With an addendum by Zak. 
[23] L. Lovász and Y. Yemini, On generic rigidity in the plane, SIAM J. Algebraic Discrete Methods 3 (1982), 91.

[24] M. J. Pflaum, Analytic and geometric study of stratified spaces, Lecture notes in mathematics, vol. 1768, Springer, 2001.

[25] A. Sard, Images of critical sets, Ann. of Math. (2) 68 (1958), no. 2, 247-259.

[26] J. B. Saxe, Embeddability of weighted graphs in k-space is strongly NP-hard, Proc. 17th Allerton Conf. in Communications, Control, and Computing, 1979, pp. 480-489.

[27] J. T. Schwartz, Fast probabilistic algorithms for verification of polynomial identities, Journal of the ACM 27 (1980), no. 4, 701-717.

[28] M. Spivak, A comprehensive introduction to differential geometry, second ed., Publish or Perish, Inc., Houston, Texas, 1979.

[29] H. Whitney, Elementary structure of real algebraic varieties, Ann. of Math. (2) 66 (1957), 545-556.

[30] F. L. Zak, Linear systems of hyperplane sections on varieties of small codimension, Funktsional. Anal. i Prilozhen 19 (1985), no. 3, 1-10.

[31] _ Tangents and secants of algebraic varieties, Translations of Mathematical Monographs, vol. 127, American Mathematical Society, Providence, RI, 1993, Translated from the Russian manuscript by the author.

[32] R. Zippel, Probabilistic algorithms for sparse polynomials, Symbolic and algebraic computation (EUROSAM '79, Internat. Sympos., Marseille, 1979), Lecture Notes in Comput. Sci., vol. 72, Springer, Berlin, 1979, pp. 216-226.

School of Engineering and Applied Sciences, Harvard University, Cambridge, MA 02138 E-mail address: sjg@cs.harvard.edu

School of Engineering and Applied Sciences, Harvard University, Cambridge, MA 02138 E-mail address: ahealy@post.harvard.edu

Department of Mathematics, Barnard College, Columbia University, New York, Ny 10027

E-mail address: dthurston@barnard.edu 